\title{
DFT Investigation of Graphene Functionalization with Activated Carbenes and its Application in the Sensing of Heavy Metallic Cations
}

Sabrine Baachaoui, ${ }^{\text {a }}$ Sarah Aldulaijan, ${ }^{\mathrm{b}}$ Luca Sementa, ${ }^{\mathrm{c}}$ Alessandro Fortunelli, ${ }^{{ }^{c} \mathrm{c}}$ Adnene Dhouib $^{* b}$ and Noureddine Raouafi*a

a Laboratory of Analytical Chemistry and Electrochemistry (LR99ES15), Chemistry department Faculty of Science of Tunis, University of Tunis El Manar, Tunis El Manar 2092, Tunisia.

b Department of Chemistry, College of Science, Imam Abdulrahman Bin Faisal University, Dammam, 31113, Saudi Arabia.

c Consiglio Nazionale delle Ricerche, CNR-ICCOM \& IPCF, 56124, Pisa, Italy

Here, for convenience of the reader, we report (1) atomistic pictures and (2) Cartesian coordinates of the structural models (all at fully relaxed geometries) derived and discussed in the main text, and additionally also (3) electron density difference plots for the absorption of the physisorption of $\mathbf{2 a}$ and $\mathbf{2} \mathbf{c}$ onto the graphene surface. 


\section{Atomistic pictures}

1.1. Geometries of relaxed $\mathrm{g} 6 \times 6 / 3 \mathrm{a}-\mathrm{M}^{2+}, 2 \mathrm{Cl}^{-}$

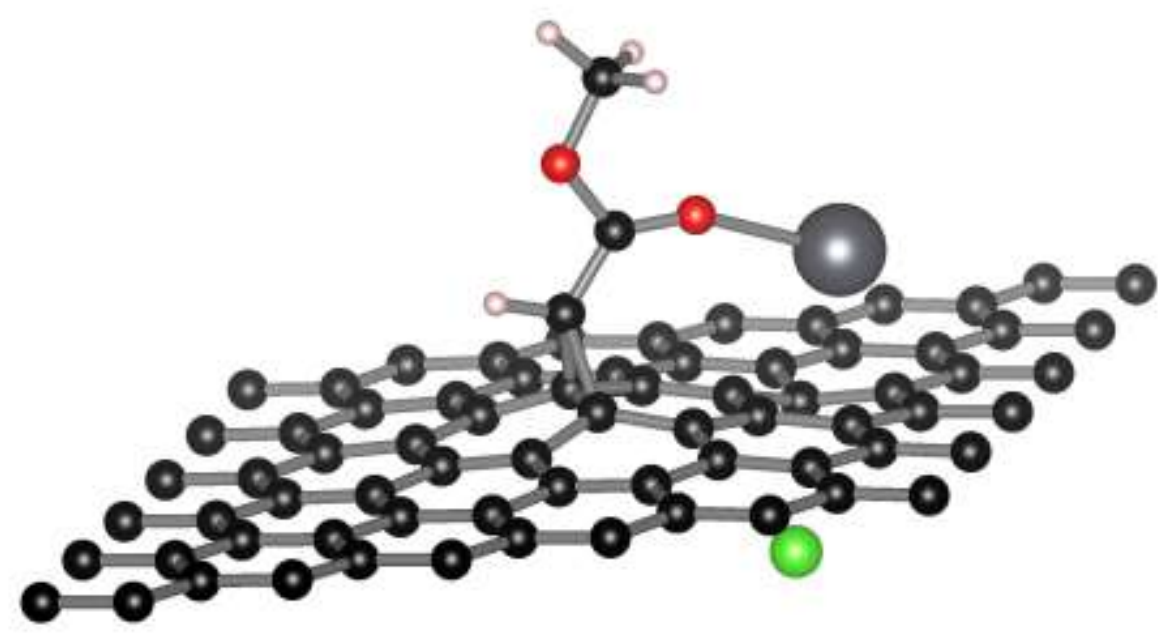

Figure S1. Final geometry of $\mathbf{g} 6 \times 6 / 3-\mathbf{P b}^{2+}, \mathbf{2} \mathrm{Cl}^{-}$(color code : C: black; 0 : red; H: White; $\mathrm{Cl}$ : green; Pb: grey)

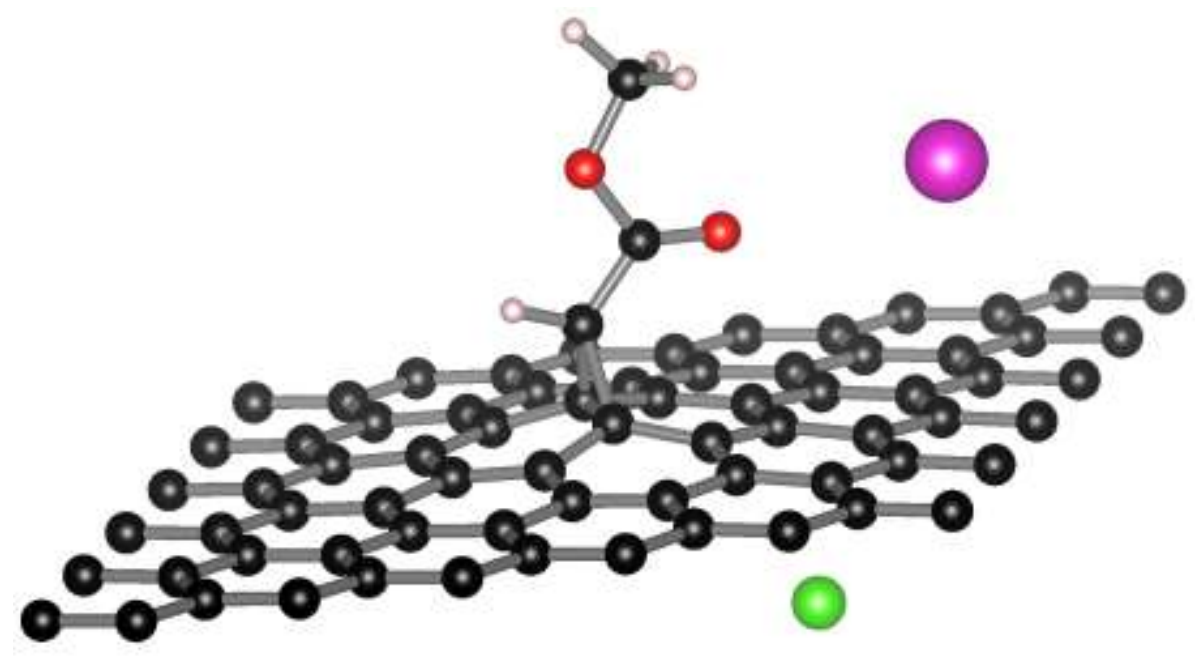

Figure S2. Final geometry of $\mathbf{g} 6 \times 6 / 3-\mathbf{C d}^{2+}, \mathbf{2} \mathbf{C l}^{-}$(color code : C: black; O : red; H: White; Cl: green; Cd: purple) 


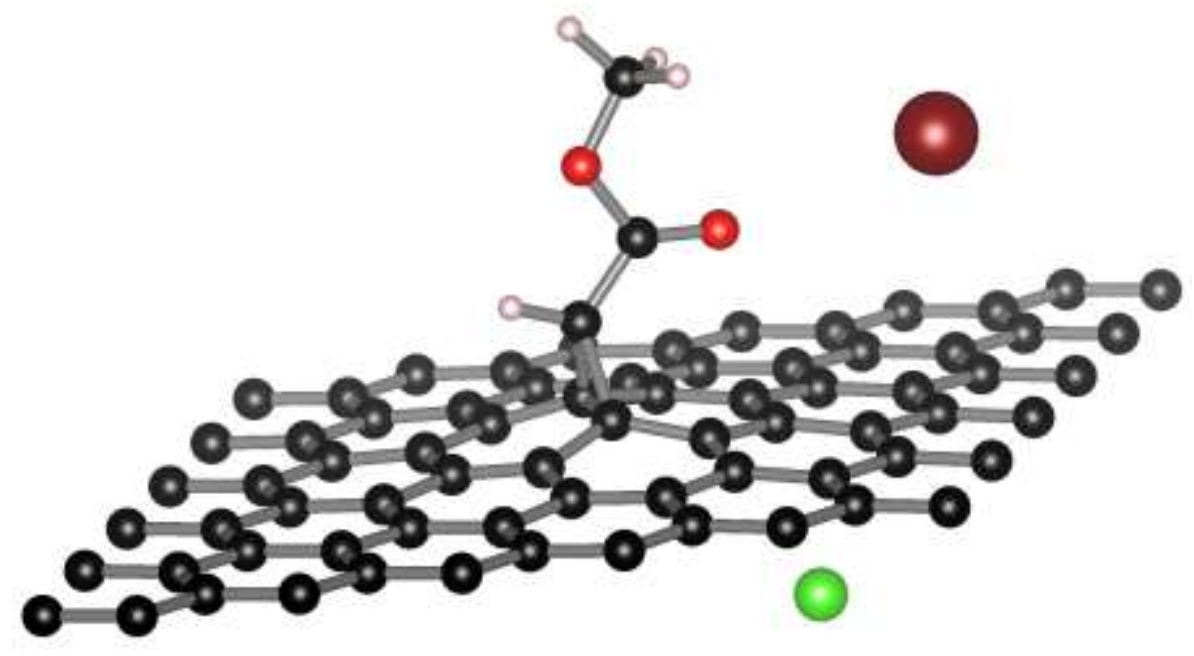

Figure S3. Final geometry of $\mathbf{g} 6 \times 6 / 3-\mathbf{H g}^{2+}, \mathbf{2} \mathrm{Cl}^{-}$(color code : C: black; $\mathrm{O}$ : red; H: White; Cl: green; Hg: brown)

1.2. Geometries of relaxed $\mathrm{g} 6 \times 6 / 3 \mathrm{~b}-\mathrm{M}^{2+}, 2 \mathrm{Cl}^{-}$

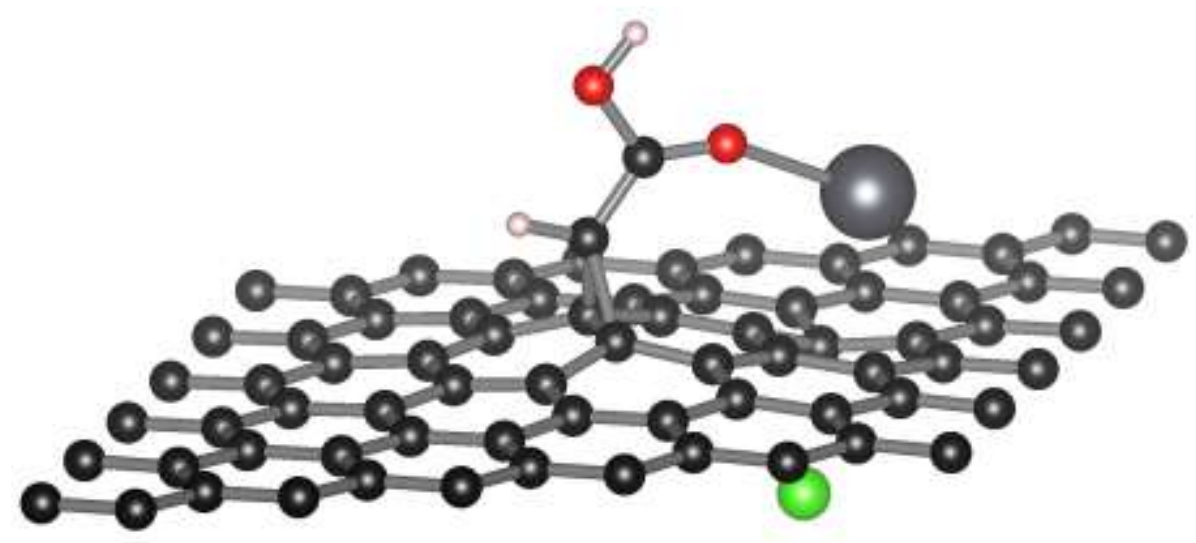

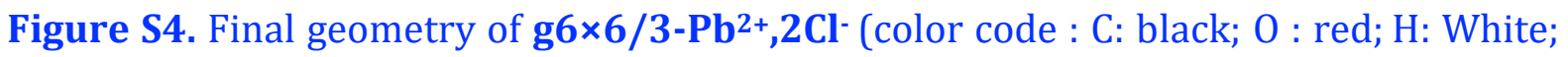
$\mathrm{Cl}$ : green; Pb: grey) 


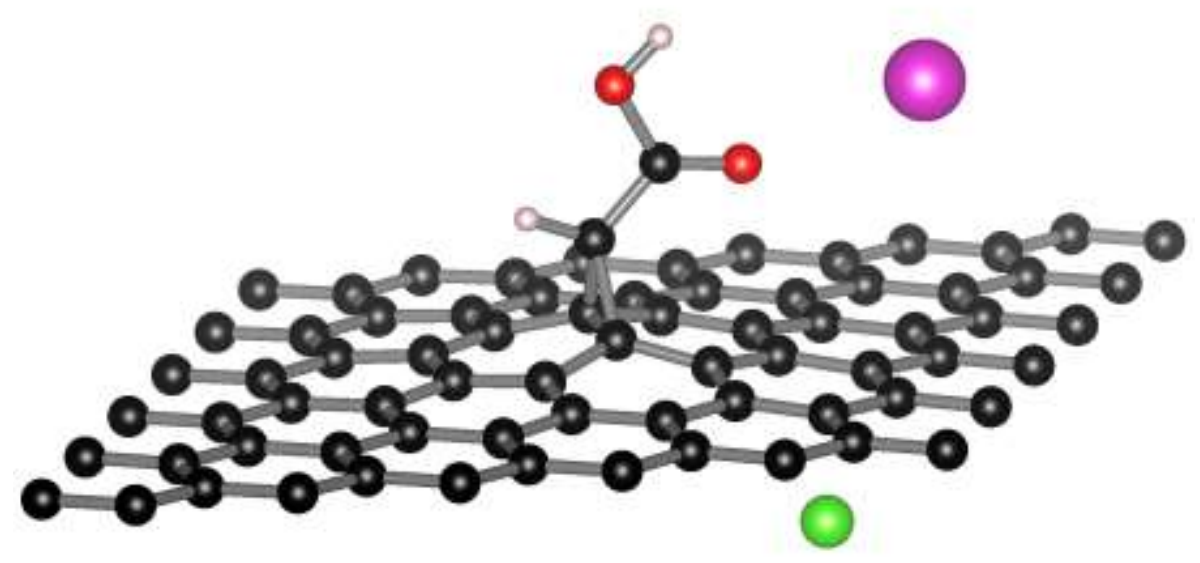

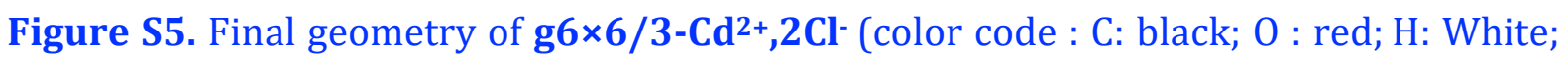
Cl: green; Cd: purple)

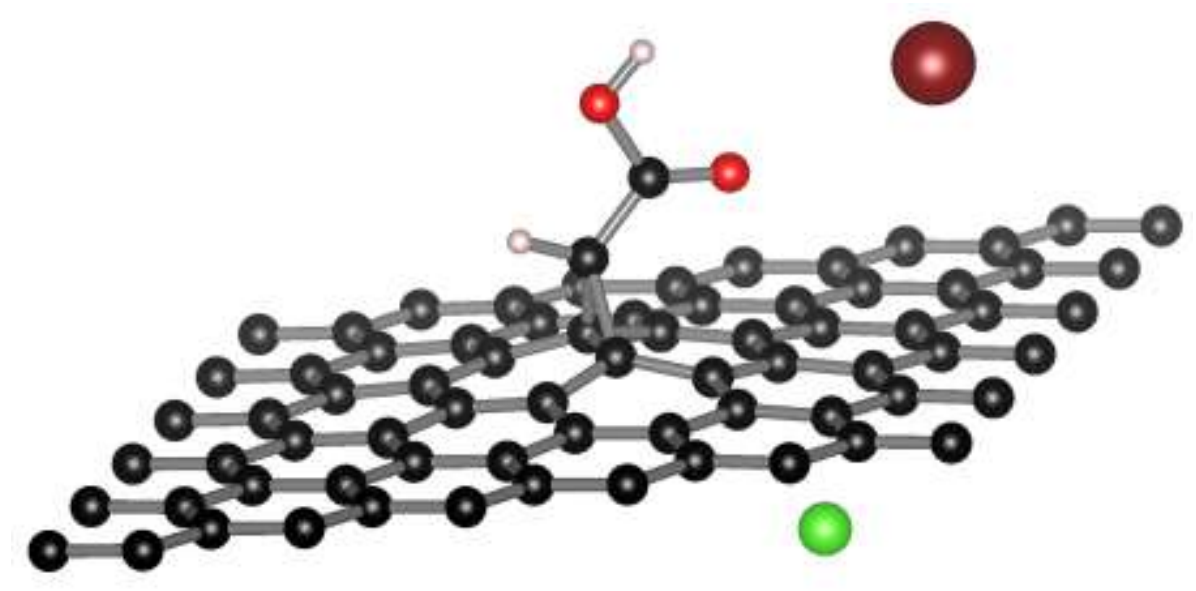

Figure S6. Final geometry of $\mathbf{g} 6 \times 6 / 3-\mathbf{H g}^{2+}, \mathbf{2} \mathrm{Cl}^{-}$(color code : C: black; 0 : red; H: White; Cl: green; Hg: bown)

\subsection{Geometries of relaxed $\mathrm{g} 6 \times 6 / 3 \mathrm{c}-\mathrm{M}^{2+}, 2 \mathrm{Cl}^{-}$}




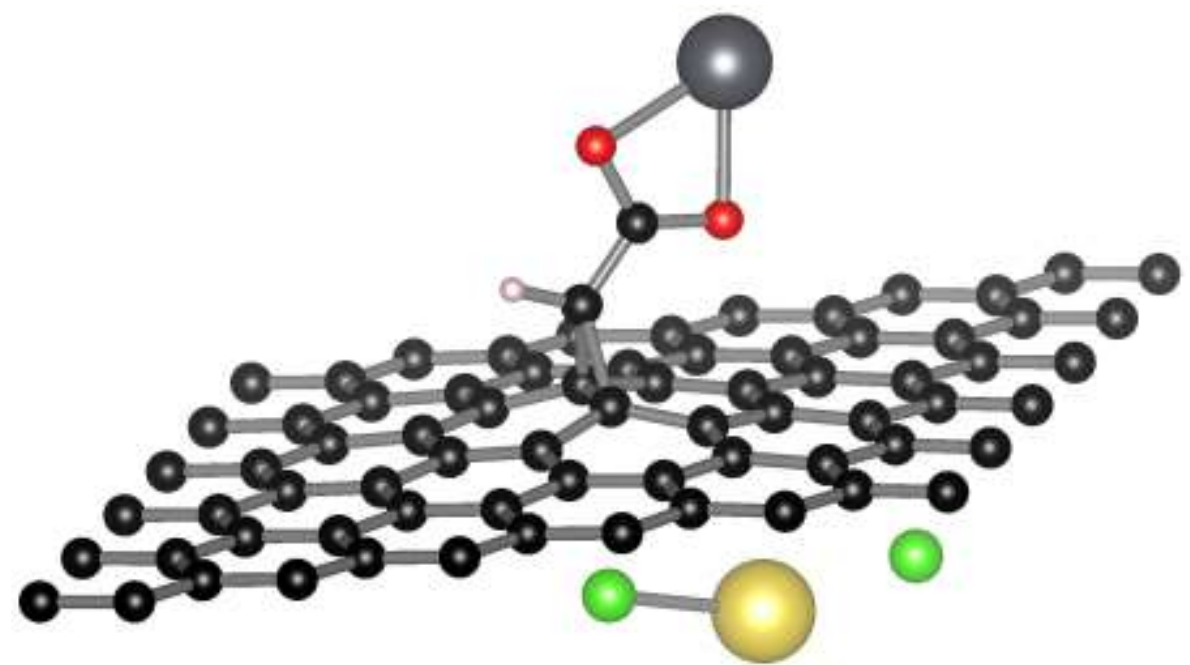

Figure S7. Final geometry of g6×6/3- $\mathrm{Pb}^{2+}, 2 \mathrm{Cl}^{-}$(color code : C: black; $\mathrm{O}$ : red; $\mathrm{H}$ : White; $\mathrm{Cl}$ : green; Na: yellow; Pb: grey)

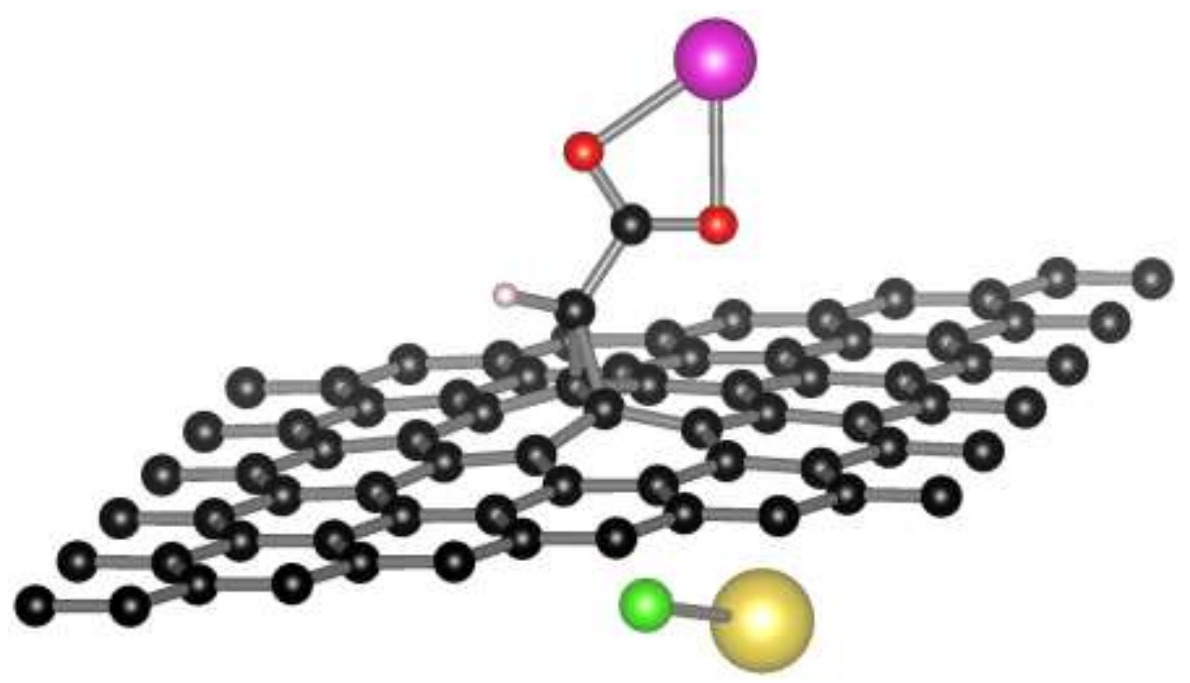

Figure S8. Final geometry of g6 $6 \times 6 / 3-\mathrm{Cd}^{2+}, 2 \mathrm{Cl}^{-}$(color code : C: black; $\mathrm{O}$ : red; $\mathrm{H}$ : White; $\mathrm{Cl}$ : green; Na: yellow; Cd: purple) 


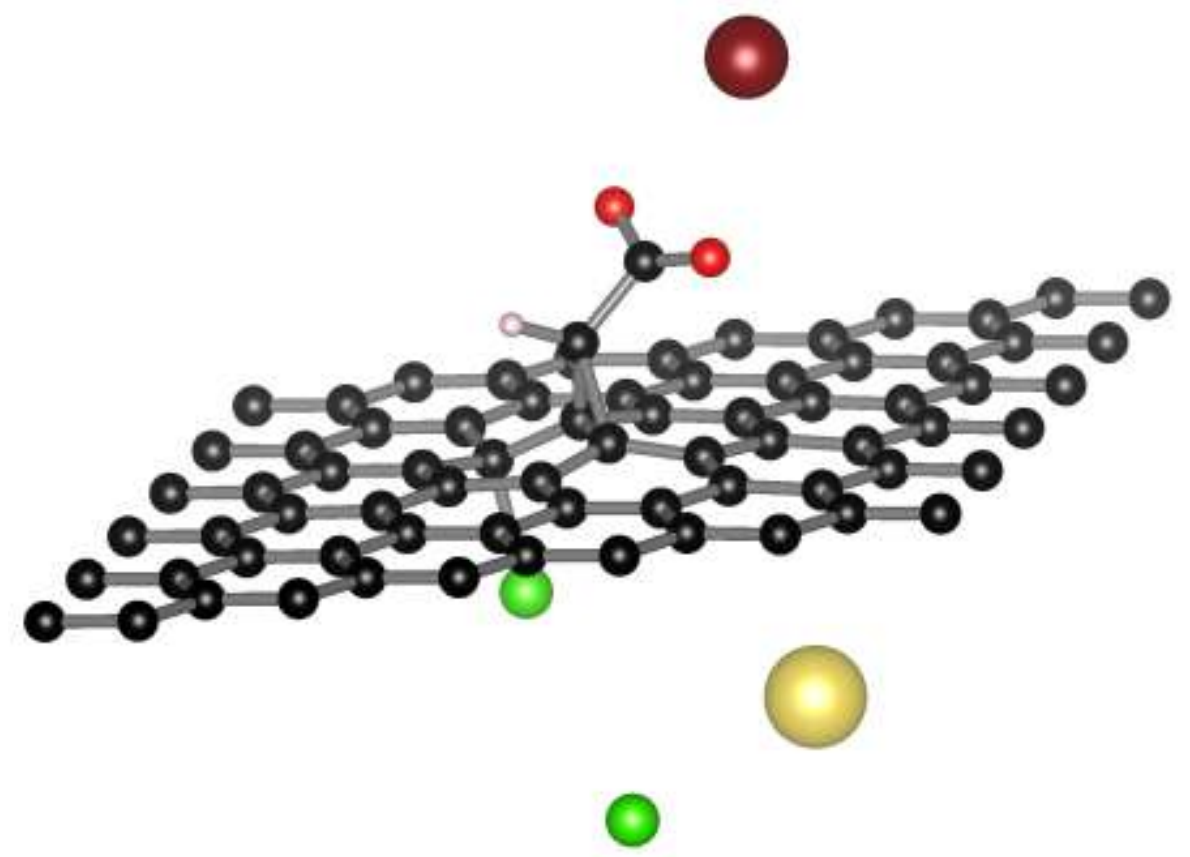

Figure S9. Final geometry of g6×6/3- $\mathrm{Hg}^{2+}, 2 \mathrm{Cl}^{-}$(color code : C: black; $\mathrm{O}$ : red; $\mathrm{H}$ : White; $\mathrm{Cl}$ : green; Na: yellow; Hg: brown)

1.4. Geometries of relaxed $\mathrm{g} 6 \times 6 / 3 \mathrm{~d}-\mathrm{M}^{2+}, 2 \mathrm{Cl}^{-}$

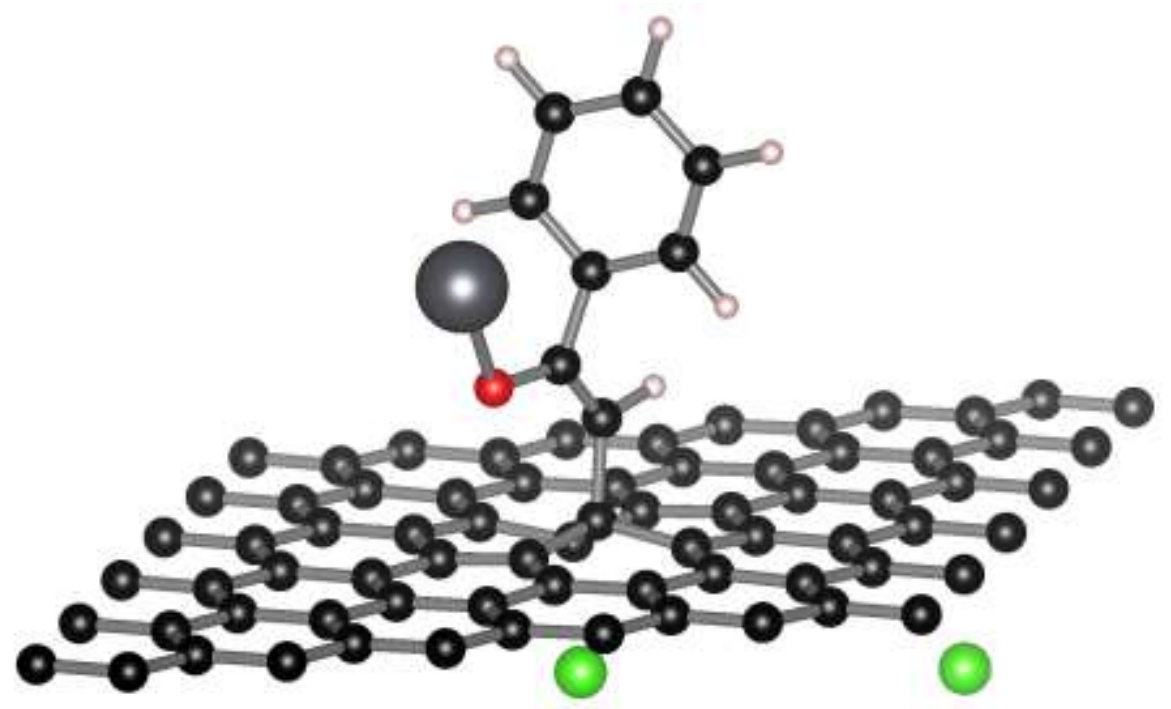

Figure S10. Final geometry of $\mathbf{g} 6 \times \mathbf{6} / \mathbf{3 d}-\mathbf{P b}^{2+}, \mathbf{2} \mathbf{C l}^{-}$(color code : C: black; O : red; H: White; $\mathrm{Cl}$ : green; Pb: grey) 


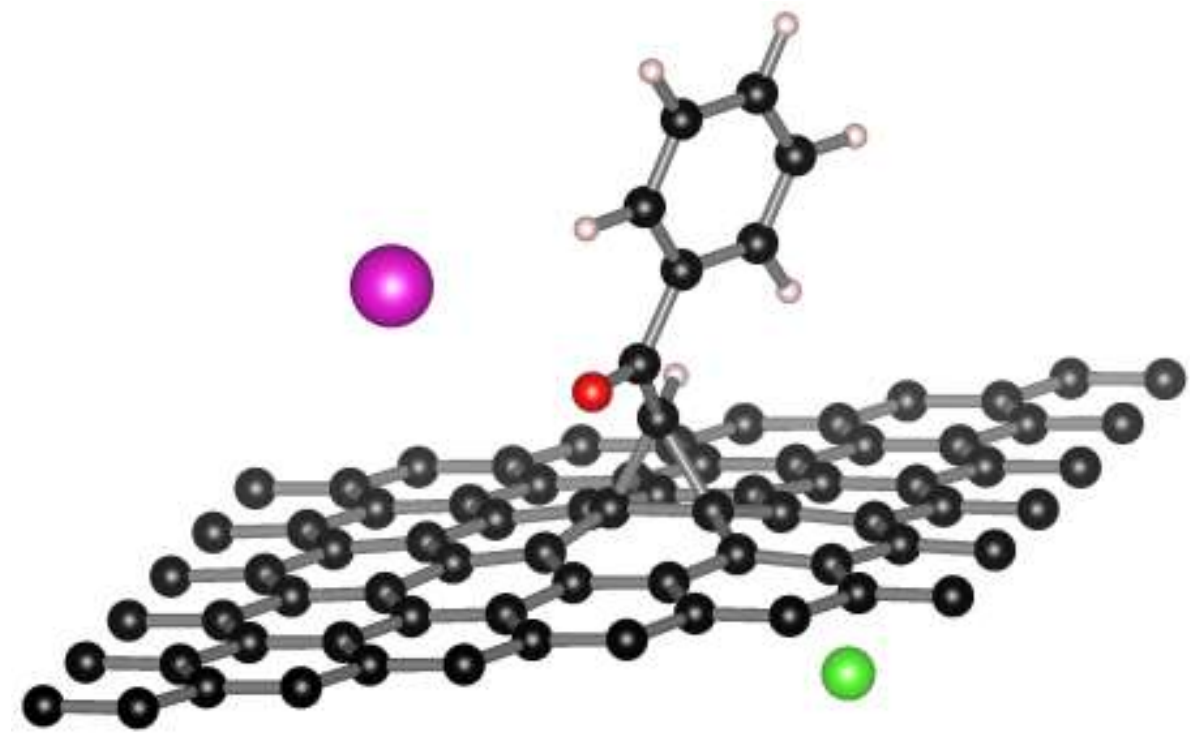

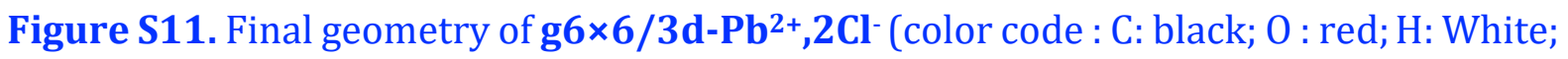
Cl: green; Cd: purple)

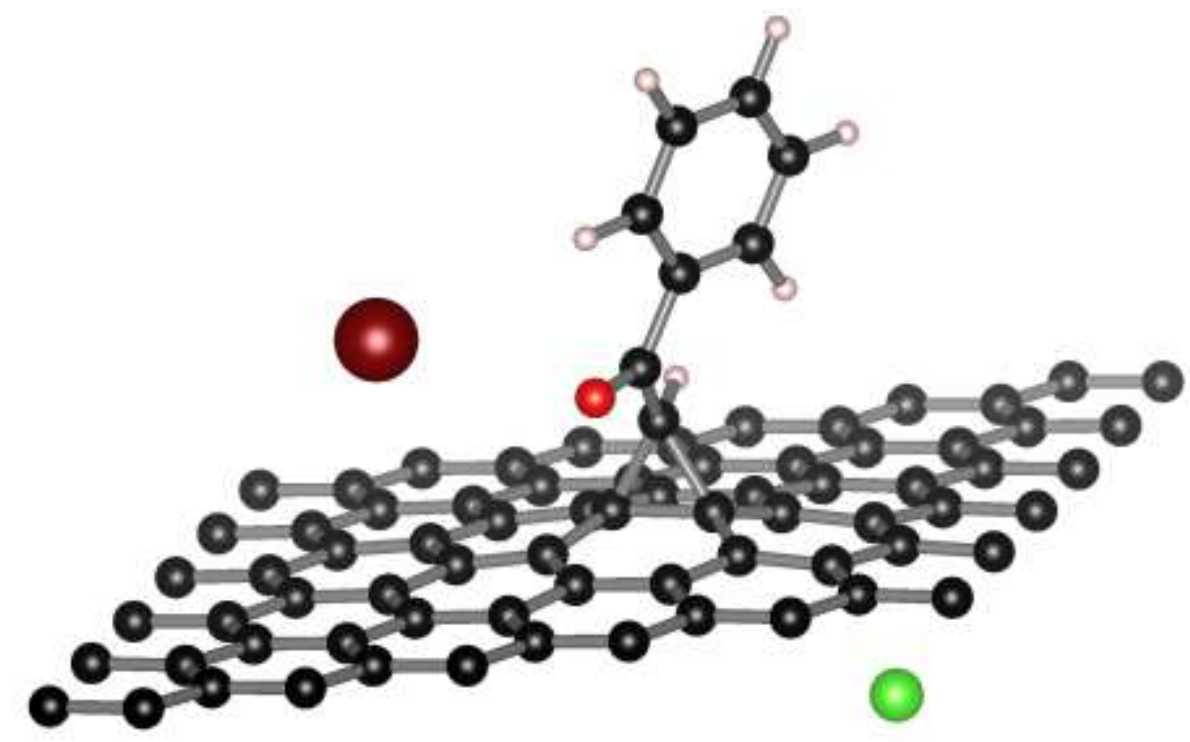

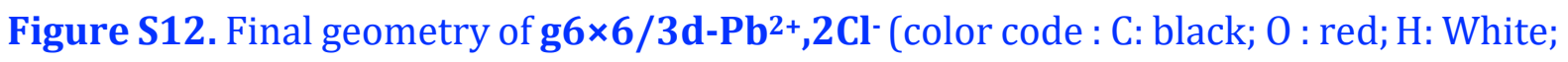
Cl: green; Hg: brown) 


\section{Supporting information}

\section{XYZ Cartesian coordinates}

\subsection{Coordinates of $\mathrm{g} 6 \times 6 / 3 \mathrm{a}-\mathrm{M}^{2+}, 2 \mathrm{Cl}^{-}$}

\subsubsection{Coordinates of $\mathrm{g} 6 \times 6 / 3 \mathrm{a}-\mathrm{Pb}^{2+}, 2 \mathrm{Cl}^{-}$}

84

\begin{tabular}{|c|c|c|}
\hline 0.0281729767 & -0.0321703348 & 7.8800431490 \\
\hline 0.0253433101 & 1.3917428578 & 7.9014165751 \\
\hline-1.2052566812 & 2.1047821278 & 7.9324517596 \\
\hline-1.2034517776 & 3.5320526577 & 7.9822350420 \\
\hline-2.4430742481 & 4.2421753929 & 7.9655446204 \\
\hline-2.4440453344 & 5.6639096737 & 7.9549433122 \\
\hline-3.6747207928 & 6.3770435894 & 7.9096738680 \\
\hline-3.6752293875 & 7.8000535052 & 7.8786749006 \\
\hline-4.9081698357 & 8.5131256389 & 7.8742886952 \\
\hline-4.9070768371 & 9.9386224030 & 7.8474322017 \\
\hline-6.1406710863 & 10.6509524751 & 7.8698023189 \\
\hline-6.1409819563 & 12.0758166298 & 7.8552302365 \\
\hline 2.4948289431 & -0.0345806293 & 7.8359116144 \\
\hline 2.4939205598 & 1.3921916181 & 7.8740001155 \\
\hline 1.2605523823 & 2.0996100383 & 7.9164705028 \\
\hline 1.2603942701 & 3.5198672819 & 8.0315467192 \\
\hline 0.0209831996 & 4.2383227745 & 8.0579934401 \\
\hline 0.0234385873 & 5.6692024410 & 8.0861808090 \\
\hline-1.2146013577 & 6.3807639528 & 7.9732166926 \\
\hline-1.2197889260 & 7.7906987246 & 7.8805714249 \\
\hline-2.4408211110 & 8.5111601834 & 7.8468832743 \\
\hline-2.4420762508 & 9.9385169575 & 7.7981230958 \\
\hline-3.6746189671 & 10.6501773091 & 7.8092903679 \\
\hline-3.6753241019 & 12.0772049389 & 7.8108732816 \\
\hline 4.9598801043 & -0.0276854890 & 7.8101311560 \\
\hline 4.9610072287 & 1.3960010691 & 7.8405882213 \\
\hline 3.7254551945 & 2.1056580945 & 7.8923774840 \\
\hline 3.7173534457 & 3.5278975561 & 8.0252254450 \\
\hline 2.4878943212 & 4.2034258710 & 8.1487190169 \\
\hline 2.4601552876 & 5.6283187722 & 8.5424902818 \\
\hline 1.2251751719 & 6.3855216774 & 8.2621291567 \\
\hline 1.2571629065 & 7.7886409840 & 8.1203426522 \\
\hline
\end{tabular}




\begin{tabular}{|c|c|c|c|}
\hline C & 0.0408604629 & 8.4916013344 & 7.7106064604 \\
\hline C & 0.0247604485 & 9.9439024898 & 7.8343034557 \\
\hline C & -1.2125934285 & 10.6548702547 & 7.8045297298 \\
\hline C & -1.2073306273 & 12.0806567884 & 7.8108983839 \\
\hline C & 7.4284922676 & -0.0253870865 & 7.8317833912 \\
\hline C & 7.4307777217 & 1.3993638191 & 7.8466917250 \\
\hline C & 6.1966473184 & 2.1091434887 & 7.8541433884 \\
\hline C & 6.1993122897 & 3.5324776726 & 7.9082990411 \\
\hline C & 4.9711507987 & 4.2500412636 & 8.0280929672 \\
\hline C & 4.9997654272 & 5.6520076977 & 8.1492264883 \\
\hline C & 3.7792036655 & 6.3850298237 & 8.5380030886 \\
\hline C & 3.7415422789 & 7.8339829528 & 8.2785969593 \\
\hline C & 2.5126520016 & 8.5109551745 & 8.1432478625 \\
\hline C & 2.5079100680 & 9.9468370470 & 7.9991223736 \\
\hline C & 1.2620153124 & 10.6632050843 & 7.8802380405 \\
\hline C & 1.2635079020 & 12.0901369499 & 7.8712104486 \\
\hline C & 9.9011678981 & -0.0229977431 & 7.8993162678 \\
\hline C & 9.9006222767 & 1.3977580042 & 7.8933284270 \\
\hline C & 8.6662458850 & 2.1082723272 & 7.8768523148 \\
\hline C & 8.6682153207 & 3.5282038258 & 7.8877842845 \\
\hline C & 7.4339329286 & 4.2412033211 & 7.9026585940 \\
\hline C & 7.4364136330 & 5.6631039164 & 7.9324978453 \\
\hline C & 6.2058369355 & 6.3737965547 & 8.0398587123 \\
\hline C & 6.2042498434 & 7.8064395593 & 8.0669343655 \\
\hline C & 4.9663089764 & 8.5228004423 & 8.1200254950 \\
\hline C & 4.9684918719 & 9.9474127981 & 8.0038306065 \\
\hline C & 3.7411170460 & 10.6629687956 & 7.9748344146 \\
\hline C & 3.7335392251 & 12.0852508358 & 7.9159073778 \\
\hline C & 12.3681067567 & -0.0262254296 & 7.9078443107 \\
\hline C & 12.3666682197 & 1.3969054685 & 7.9150963735 \\
\hline C & 11.1329060753 & 2.1074393714 & 7.9068023713 \\
\hline C & 11.1330648945 & 3.5312088060 & 7.9263055907 \\
\hline C & 9.8995494112 & 4.2405610516 & 7.9072169388 \\
\hline C & 9.8982638208 & 5.6630416020 & 7.9054511654 \\
\hline C & 8.6671410897 & 6.3774681542 & 7.9111597320 \\
\hline C & 8.6653603982 & 7.8006628572 & 7.9144247670 \\
\hline C & 7.4282368871 & 8.5139349536 & 7.9657436546 \\
\hline
\end{tabular}




$\begin{array}{llll}\mathrm{C} & 7.4317016187 & 9.9415418858 & 7.9279019864 \\ \mathrm{C} & 6.2009755783 & 10.6550750420 & 7.9399324348 \\ \mathrm{C} & 6.1974219538 & 12.0795882540 & 7.9045559335 \\ \mathrm{C} & 3.2209142417 & 5.8276251815 & 9.9138256351 \\ \mathrm{C} & 2.8208658838 & 6.5736317536 & 11.0787931924 \\ \mathrm{H} & 3.7369488104 & 4.9137398366 & 10.1996853758 \\ \mathrm{O} & 3.1603133988 & 5.9055497646 & 12.2071240955 \\ \mathrm{O} & 2.2587530131 & 7.6898323690 & 11.1729043534 \\ \mathrm{C} & 2.8559801875 & 6.5608274773 & 13.4595263403 \\ \mathrm{~Pb} & 1.0128043826 & 9.5237957936 & 10.3035019653 \\ \mathrm{Cl} & 10.5024395737 & 2.2802405026 & 4.8167524995 \\ \mathrm{Cl} & 0.2762392051 & 8.3237033455 & 5.4524218192 \\ \mathrm{H} & 3.1970139496 & 5.8704145029 & 14.2354488443 \\ \mathrm{H} & 1.7764467162 & 6.7357549967 & 13.5490366153 \\ \mathrm{H} & 3.3920289475 & 7.5157643249 & 13.5304200652\end{array}$

\subsubsection{Coordinates of $\mathrm{g} 6 \times 6 / 3 \mathrm{a}-\mathrm{Cd}^{2+}, 2 \mathrm{Cl}^{-}$}

84

$\begin{array}{llll}\text { C } & 0.0317198225 & -0.0567717143 & 7.8609621055 \\ \text { C } & 0.0315717704 & 1.3670660701 & 7.8752076861 \\ \text { C } & -1.2004734252 & 2.0809760091 & 7.8935268310 \\ \text { C } & -1.1976846762 & 3.5091391544 & 7.9413360053 \\ \text { C } & -2.4357514994 & 4.2197454859 & 7.9186919517 \\ \text { C } & -2.4355161445 & 5.6436621691 & 7.9275155980 \\ \text { C } & -3.6679055971 & 6.3551650272 & 7.8913817638 \\ \text { C } & -3.6678136223 & 7.7790302450 & 7.8923489038 \\ \text { C } & -4.9033222906 & 8.4901114282 & 7.8785571494 \\ \text { C } & -4.9025400543 & 9.9150557930 & 7.8689459091 \\ \text { C } & -6.1375269042 & 10.6266249098 & 7.8680466881 \\ \text { C } & -6.1360905267 & 12.0518094128 & 7.8538768767 \\ \text { C } & 2.5005264151 & -0.0591567945 & 7.8545063604 \\ \text { C } & 2.4998581179 & 1.3671236711 & 7.8841250723 \\ \text { C } & 1.2663130566 & 2.0748389044 & 7.9014682847 \\ \text { C } & 1.2666354629 & 3.4965798603 & 8.0118699451 \\ \text { C } & 0.0270995806 & 4.2182104829 & 8.0300069249 \\ \text { C } & 0.0251648977 & 5.6498226329 & 8.0858527744 \\ \text { C } & -1.2069157815 & 6.3595913094 & 7.9919455748\end{array}$




\begin{tabular}{|c|c|c|c|}
\hline C & -1.2031750916 & 7.7798044950 & 7.9878760989 \\
\hline C & -2.4350937307 & 8.4916182030 & 7.9242303005 \\
\hline C & -2.4331841083 & 9.9147584204 & 7.9000282300 \\
\hline C & -3.6672618400 & 10.6262092023 & 7.8679341425 \\
\hline C & -3.6675827524 & 12.0518006051 & 7.8595639664 \\
\hline C & 4.9690147515 & -0.0555213708 & 7.8664619706 \\
\hline C & 4.9686336043 & 1.3691054232 & 7.8833646929 \\
\hline C & 3.7320495738 & 2.0804324358 & 7.9214830362 \\
\hline C & 3.7239563411 & 3.5017315032 & 8.0497688881 \\
\hline C & 2.4916516637 & 4.1787587523 & 8.1503405670 \\
\hline C & 2.4539315030 & 5.6007595597 & 8.5402848160 \\
\hline C & 1.2308195183 & 6.3693504222 & 8.2527450439 \\
\hline C & 1.2575641488 & 7.7726858888 & 8.1677857444 \\
\hline C & 0.0316548648 & 8.4881426835 & 8.0263416597 \\
\hline C & 0.0339278663 & 9.9144780060 & 7.9711386437 \\
\hline C & -1.1974708394 & 10.6257633102 & 7.9141066267 \\
\hline C & -1.1982881001 & 12.0515012132 & 7.8837975377 \\
\hline C & 7.4364667299 & -0.0571946364 & 7.8850282605 \\
\hline C & 7.4371600459 & 1.3688840168 & 7.8777699952 \\
\hline C & 6.2037328340 & 2.0814744363 & 7.8905091226 \\
\hline C & 6.2052833721 & 3.5075026430 & 7.9350317975 \\
\hline C & 4.9772931228 & 4.2250767495 & 8.0586103129 \\
\hline C & 5.0058552916 & 5.6304113692 & 8.1690373220 \\
\hline C & 3.7893144369 & 6.3748841706 & 8.5524822842 \\
\hline C & 3.7374908953 & 7.8178319354 & 8.2569193769 \\
\hline C & 2.5076592482 & 8.4958714321 & 8.1658851303 \\
\hline C & 2.5017786227 & 9.9157402870 & 8.0277268979 \\
\hline C & 1.2669475075 & 10.6273873643 & 7.9719160299 \\
\hline C & 1.2679043295 & 12.0505684299 & 7.9159569964 \\
\hline C & 9.9027433948 & -0.0544275321 & 7.9055297924 \\
\hline C & 9.9038384739 & 1.3688256751 & 7.8797673913 \\
\hline C & 8.6711791740 & 2.0805090575 & 7.8800485751 \\
\hline C & 8.6736334614 & 3.5031837509 & 7.8797464123 \\
\hline C & 7.4397434830 & 4.2165637457 & 7.9086760721 \\
\hline C & 7.4420467531 & 5.6385667375 & 7.9234827648 \\
\hline C & 6.2104451136 & 6.3492359537 & 8.0325426106 \\
\hline C & 6.2045893748 & 7.7830722613 & 8.0429916485 \\
\hline
\end{tabular}




$\begin{array}{lccc}\mathrm{C} & 4.9645705612 & 8.5015127370 & 8.0916315437 \\ \mathrm{C} & 4.9657413652 & 9.9228271927 & 7.9852876260 \\ \mathrm{C} & 3.7319122892 & 10.6307192906 & 7.9784353086 \\ \mathrm{C} & 3.7328061888 & 12.0559185122 & 7.9184972758 \\ \mathrm{C} & 12.3683070904 & -0.0541884803 & 7.8869756146 \\ \mathrm{C} & 12.3709417157 & 1.3710176364 & 7.8795975376 \\ \mathrm{C} & 11.1378145523 & 2.0821405584 & 7.8770908497 \\ \mathrm{C} & 11.1390226016 & 3.5065164526 & 7.8856884852 \\ \mathrm{C} & 9.9047280841 & 4.2166494632 & 7.8764763122 \\ \mathrm{C} & 9.9047248665 & 5.6403611142 & 7.8770487534 \\ \mathrm{C} & 8.6719759368 & 6.3533967757 & 7.8899675328 \\ \mathrm{C} & 8.6688116774 & 7.7771666259 & 7.8975264726 \\ \mathrm{C} & 7.4301363625 & 8.4896757174 & 7.9440589834 \\ \mathrm{C} & 7.4323304075 & 9.9176162621 & 7.9075658103 \\ \mathrm{C} & 6.1995600304 & 10.6303425978 & 7.9168125265 \\ \mathrm{C} & 6.1992539644 & 12.0542790609 & 7.8821009121 \\ \mathrm{C} & 3.1934151029 & 5.8488340546 & 9.8817138896 \\ \mathrm{C} & 2.6679642827 & 6.6541482147 & 11.0094275239 \\ \mathrm{H} & 3.7080466023 & 4.9561230885 & 10.2316987080 \\ \mathrm{O} & 2.9624802557 & 5.9835474529 & 12.1628742847 \\ \mathrm{O} & 2.0649996594 & 7.7142780028 & 10.9758567428 \\ \mathrm{C} & 2.4865776377 & 6.6033271610 & 13.3804204086 \\ \mathrm{H} & 2.9759588171 & 7.5735683358 & 13.5335495781 \\ \mathrm{H} & 1.4000367015 & 6.7495745281 & 13.3353647625 \\ \mathrm{H} & 2.7511516427 & 5.9067568455 & 14.1813122202 \\ \mathrm{Cl} & 1.7442273446 & 11.0940186553 & 11.9602892733 \\ \mathrm{C} & 10.3121518400 & 2.1013243188 & 4.7426169793 \\ \mathrm{H} & 0.2852727797 & 8.3097601684 & 4.9147579207\end{array}$

\subsubsection{Coordinates of $\mathrm{g} 6 \times 6 / 3 \mathrm{~b}-\mathrm{Hg}^{2+}, 2 \mathrm{Cl}^{-}$}

84

$\begin{array}{llll}\text { C } & 0.0396954013 & -0.0602456873 & 7.8620761002 \\ \text { C } & 0.0395212902 & 1.3638475516 & 7.8771973466 \\ \text { C } & -1.1926574781 & 2.0775924577 & 7.8989388755 \\ \text { C } & -1.1902403308 & 3.5058164272 & 7.9460957323 \\ \text { C } & -2.4281920904 & 4.2166152987 & 7.9247000652 \\ \text { C } & -2.4277175577 & 5.6407251227 & 7.9311038057\end{array}$




\begin{tabular}{|c|c|c|c|}
\hline C & -3.6598777856 & 6.3518077678 & 7.8907821190 \\
\hline C & -3.6596769948 & 7.7760193292 & 7.8903591277 \\
\hline C & -4.8951635312 & 8.4868750994 & 7.8732516365 \\
\hline C & -4.8940075274 & 9.9120657970 & 7.8677717170 \\
\hline C & -6.1293522850 & 10.6231641548 & 7.8672355990 \\
\hline C & -6.1279545898 & 12.0484873237 & 7.8551362223 \\
\hline C & 2.5086683083 & -0.0621897446 & 7.8557966971 \\
\hline C & 2.5078721631 & 1.3641286074 & 7.8837471245 \\
\hline C & 1.2742684529 & 2.0719523850 & 7.9008598188 \\
\hline C & 1.2746243240 & 3.4939115601 & 8.0096672365 \\
\hline C & 0.0346081378 & 4.2151542874 & 8.0331342387 \\
\hline C & 0.0323577778 & 5.6461997851 & 8.0951107640 \\
\hline C & -1.1993552220 & 6.3563491476 & 7.9995858947 \\
\hline C & -1.1956901639 & 7.7764815028 & 7.9954510299 \\
\hline C & -2.4270269882 & 8.4883656291 & 7.9276981957 \\
\hline C & -2.4250899082 & 9.9117637169 & 7.9027961128 \\
\hline C & -3.6588222184 & 10.6233340353 & 7.8688955641 \\
\hline C & -3.6590977383 & 12.0488685146 & 7.8589109807 \\
\hline C & 4.9776728482 & -0.0586922865 & 7.8627981547 \\
\hline C & 4.9772514923 & 1.3657676598 & 7.8773720243 \\
\hline C & 3.7404118879 & 2.0773440471 & 7.9152082287 \\
\hline C & 3.7324678555 & 3.4988149787 & 8.0399796304 \\
\hline C & 2.5001360926 & 4.1759864713 & 8.1454987672 \\
\hline C & 2.4633384193 & 5.5959811018 & 8.5419882162 \\
\hline C & 1.2385084092 & 6.3656197010 & 8.2648028138 \\
\hline C & 1.2655543732 & 7.7690646945 & 8.1827903227 \\
\hline C & 0.0399870819 & 8.4842128671 & 8.0346259645 \\
\hline C & 0.0418923734 & 9.9110095158 & 7.9762352807 \\
\hline C & -1.1892807888 & 10.6225031435 & 7.9163943245 \\
\hline C & -1.1898115425 & 12.0480099271 & 7.8818027675 \\
\hline C & 7.4448810415 & -0.0607500721 & 7.8866471564 \\
\hline C & 7.4459320558 & 1.3652672501 & 7.8770984115 \\
\hline C & 6.2124825425 & 2.0779096045 & 7.8832299126 \\
\hline C & 6.2140669400 & 3.5038291901 & 7.9227838438 \\
\hline C & 4.9860778661 & 4.2216857130 & 8.0450478994 \\
\hline C & 5.0147398898 & 5.6263129088 & 8.1568665649 \\
\hline C & 3.8003136456 & 6.3685245568 & 8.5528555958 \\
\hline
\end{tabular}




\begin{tabular}{|c|c|c|c|}
\hline C & 3.7463399916 & 7.8128050583 & 8.2611782352 \\
\hline C & 2.5164592704 & 8.4916098921 & 8.1749493511 \\
\hline C & 2.5098574492 & 9.9115562918 & 8.0350952652 \\
\hline C & 1.2748814416 & 10.6231022025 & 7.9762607069 \\
\hline C & 1.2759102781 & 12.0463778309 & 7.9195249442 \\
\hline C & 9.9106587864 & -0.0585242671 & 7.9115656538 \\
\hline C & 9.9124654097 & 1.3646128178 & 7.8879268787 \\
\hline C & 8.6800574608 & 2.0764591536 & 7.8828982639 \\
\hline C & 8.6822482043 & 3.4990656493 & 7.8780924479 \\
\hline C & 7.4485225638 & 4.2125719977 & 7.8969710835 \\
\hline C & 7.4507356185 & 5.6346252333 & 7.9079404009 \\
\hline C & 6.2188039787 & 6.3453420242 & 8.0171712492 \\
\hline C & 6.2123686340 & 7.7790527854 & 8.0304763061 \\
\hline C & 4.9721556942 & 8.4966502024 & 8.0860644311 \\
\hline C & 4.9731478121 & 9.9185283337 & 7.9820231753 \\
\hline C & 3.7397874011 & 10.6266287776 & 7.9810751068 \\
\hline C & 3.7405995729 & 12.0518391853 & 7.9221140386 \\
\hline C & 12.3762888907 & -0.0579542972 & 7.8911660089 \\
\hline C & 12.3790052431 & 1.3671865828 & 7.8894076124 \\
\hline C & 11.1461986955 & 2.0784043996 & 7.8883713698 \\
\hline C & 11.1470566621 & 3.5028367116 & 7.8935116850 \\
\hline C & 9.9130624975 & 4.2127428806 & 7.8782473288 \\
\hline C & 9.9130718068 & 5.6367004977 & 7.8733618300 \\
\hline C & 8.6805660219 & 6.3497758588 & 7.8786254035 \\
\hline C & 8.6770529998 & 7.7738030335 & 7.8877384210 \\
\hline C & 7.4379513441 & 8.4859583277 & 7.9345975298 \\
\hline C & 7.4403645510 & 9.9140697059 & 7.9036615152 \\
\hline C & 6.2073571317 & 10.6262639051 & 7.9136470553 \\
\hline C & 6.2069826535 & 12.0504273137 & 7.8818211091 \\
\hline C & 3.2116405909 & 5.8360480870 & 9.8813128888 \\
\hline C & 2.7044972842 & 6.6448290339 & 11.0160574926 \\
\hline $\mathrm{H}$ & 3.7196466761 & 4.9371971467 & 10.2262254134 \\
\hline $\mathrm{O}$ & 2.9435892686 & 5.9359648593 & 12.1599094452 \\
\hline $\mathrm{O}$ & 2.1630643425 & 7.7370553137 & 10.9940416954 \\
\hline C & 2.5051275212 & 6.5628753932 & 13.3874337857 \\
\hline $\mathrm{H}$ & 3.0320949830 & 7.5123620076 & 13.5431698817 \\
\hline & 1.4239430517 & 6.7469833820 & 13.361276 \\
\hline
\end{tabular}




$\begin{array}{llll}\mathrm{H} & 2.7543516648 & 5.8520582348 & 14.1803322727 \\ \mathrm{Hg} & -0.0270967006 & 10.5132053314 & 11.8590717553 \\ \mathrm{Cl} & 10.3050698624 & 2.1227932564 & 4.7517731555 \\ \mathrm{Cl} & 0.2243194610 & 8.3558038237 & 4.9553135197\end{array}$

\subsection{Coordinates of $\mathrm{g} 6 \times 6 / 3 \mathrm{~b}-\mathrm{M}^{2+}, 2 \mathrm{Cl}^{-}$}

\subsubsection{Coordinates of $\mathrm{g} 6 \times 6 / 3 \mathrm{~b}-\mathrm{Pb}^{2+}, 2 \mathrm{Cl}^{-}$}

81

$\begin{array}{llll}\text { C } & 0.0274843056 & -0.0299989195 & 7.8952386138 \\ \text { C } & 0.0252093088 & 1.3940022621 & 7.9176842975 \\ \text { C } & -1.2054859224 & 2.1071226596 & 7.9480838296 \\ \text { C } & -1.2033436994 & 3.5344980405 & 7.9932613367 \\ \text { C } & -2.4431536649 & 4.2452029475 & 7.9753174113 \\ \text { C } & -2.4438670025 & 5.6670386187 & 7.9569529435 \\ \text { C } & -3.6748032888 & 6.3799633859 & 7.9148877825 \\ \text { C } & -3.6753129630 & 7.8028040798 & 7.8788949902 \\ \text { C } & -4.9084661283 & 8.5158158561 & 7.8845349197 \\ \text { C } & -4.9075953313 & 9.9411173590 & 7.8537956070 \\ \text { C } & -6.1413515320 & 10.6532516423 & 7.8838516588 \\ \text { C } & -6.1415197729 & 12.0781262651 & 7.8682759550 \\ \text { C } & 2.4945065161 & -0.0325319187 & 7.8504528771 \\ \text { C } & 2.4940509274 & 1.3941527936 & 7.8975829697 \\ \text { C } & 1.2600380952 & 2.1013491994 & 7.9376141838 \\ \text { C } & 1.2598527259 & 3.5217057839 & 8.0516662807 \\ \text { C } & 0.0206657914 & 4.2399778557 & 8.0681043583 \\ \text { C } & 0.0243636947 & 5.6713807230 & 8.0887860722 \\ \text { C } & -1.2141131012 & 6.3838407605 & 7.9664420572 \\ \text { C } & -1.2195042824 & 7.7919587868 & 7.8619847241 \\ \text { C } & -2.4401707938 & 8.5131031235 & 7.8329941225 \\ \text { C } & -2.4421696568 & 9.9409591540 & 7.7840727713 \\ \text { C } & -3.6746387194 & 10.6522304495 & 7.8064074887 \\ \text { C } & -3.6754102747 & 12.0797577940 & 7.8176167985 \\ \text { C } & 4.9598855864 & -0.0255147034 & 7.8226203870 \\ \text { C } & 4.9610075426 & 1.3980617969 & 7.8692536803 \\ \text { C } & 3.7255068683 & 2.1072979346 & 7.9239292422 \\ \text { C.7172055404 } & 3.5294389800 & 8.0597319953\end{array}$




$\begin{array}{llll}\text { C } & 2.4866803694 & 4.2052308598 & 8.1741712763 \\ \text { C } & 2.4579891943 & 5.6312148675 & 8.5637484497 \\ \text { C } & 1.2230304250 & 6.3867715904 & 8.2761802144 \\ \text { C } & 1.2547649265 & 7.7927759244 & 8.1318707133 \\ \text { C } & 0.0469774375 & 8.4905234092 & 7.6630734575 \\ \text { C } & 0.0262247810 & 9.9461070544 & 7.8228294346 \\ \text { C } & -1.2113925962 & 10.6564493061 & 7.7940370087 \\ \text { C } & -1.2069710889 & 12.0828665871 & 7.8159564601 \\ \text { C } & 7.4285103624 & -0.0230091133 & 7.8473535225 \\ \text { C } & 7.4303706242 & 1.4019582908 & 7.8740444514 \\ \text { C } & 6.1965490092 & 2.1114884070 & 7.8892922761 \\ \text { C } & 6.1989832784 & 3.5345039973 & 7.9519385754 \\ \text { C } & 4.9708154138 & 4.2518240437 & 8.0692442539 \\ \text { C } & 4.9987680326 & 5.6545573303 & 8.1884173165 \\ \text { C } & 12.3662606606 & 1.3994885383 & 7.9343316369 \\ \text { C } & 3.7748930977 & 6.3864694080 & 8.5645488185 \\ \text { C } & 3.7402418269 & 7.8363710302 & 8.3137470421 \\ \text { C } & 11.1327606263 & 2.1102861705 & 7.9270711599 \\ \text { C } & 2.5113065607 & 8.5143680486 & 8.1668377224 \\ \text { C } & 2.5069131208 & 9.9503403453 & 8.0211083372 \\ \text { C } & 1.1329110131 & 3.5344674099 & 7.9432361006 \\ \text { C } & 1.2615508175 & 10.6650371472 & 7.8910118973 \\ \text { C } & 1.2630923858 & 12.0921939019 & 7.8898203777 \\ \text { C } & 9.9006605142 & -0.0203646791 & 7.9249604879 \\ \text { C } & 9.8999909370 & 1.4003108919 & 7.9182565028 \\ \text { C } & 8.6659426221 & 2.1111740323 & 7.9056518082 \\ \text { C } & 8.6675494441 & 3.5311706407 & 7.9202791919 \\ \text { C } & 7.4336160408 & 4.2439508104 & 7.9443287097 \\ \text { C } & 7.4357735917 & 5.6661621284 & 7.9734112510 \\ \text { C } & 12.3676105329 & -0.0234377770 & 7.9306632330 \\ \text { C } & 3.2438456820 & 7.9299910631\end{array}$




$\begin{array}{lrrr}\mathrm{C} & 9.8976597405 & 5.6662208330 & 7.9243277974 \\ \mathrm{C} & 8.6666554650 & 6.3803743476 & 7.9406317086 \\ \mathrm{C} & 8.6647226050 & 7.8036575752 & 7.9393058811 \\ \mathrm{C} & 7.4278755619 & 8.5166774068 & 7.9966246669 \\ \mathrm{C} & 7.4310523156 & 9.9440001134 & 7.9519032614 \\ \mathrm{C} & 6.2000624709 & 10.6576106591 & 7.9658436924 \\ \mathrm{C} & 6.1966917012 & 12.0818435011 & 7.9244022661 \\ \mathrm{C} & 3.2130305312 & 5.8179978733 & 9.9440282950 \\ \mathrm{C} & 2.8214456324 & 6.5478299878 & 11.1132060733 \\ \mathrm{H} & 3.7330472579 & 4.9027281624 & 10.2169293859 \\ \mathrm{O} & 3.1888983941 & 5.8882715206 & 12.2446733570 \\ \mathrm{O} & 2.2387352798 & 7.6527310660 & 11.2404871129 \\ \mathrm{H} & 2.9153992890 & 6.4456323109 & 13.0016020877 \\ \mathrm{~Pb} & 0.9711461410 & 9.4452646181 & 10.2994011064 \\ \mathrm{Cl} & 10.5184177155 & 2.2792778497 & 4.8430583522 \\ \mathrm{Cl} & 0.2919012385 & 8.3067092945 & 5.4875330657\end{array}$

\subsubsection{Coordinates of $\mathrm{g} 6 \times 6 / 3 \mathrm{~b}-\mathrm{Cd}^{2+}, 2 \mathrm{Cl}^{-}$}

81

$\begin{array}{llll}\text { C } & 0.0414722948 & -0.0549064330 & 7.8753005870 \\ \text { C } & 0.0406654015 & 1.3687346166 & 7.9052411327 \\ \text { C } & -1.1914257958 & 2.0824019105 & 7.9240647920 \\ \text { C } & -1.1891552444 & 3.5101458934 & 7.9735501408 \\ \text { C } & -2.4271103364 & 4.2207348067 & 7.9456134985 \\ \text { C } & -2.4268470860 & 5.6444090809 & 7.9419790475 \\ \text { C } & -3.6587823070 & 6.3559492402 & 7.9032728086 \\ \text { C } & -3.6582981013 & 7.7801291733 & 7.8909405943 \\ \text { C } & -4.8937105662 & 8.4909956887 & 7.8780100065 \\ \text { C } & -4.8929869312 & 9.9162681312 & 7.8652058620 \\ \text { C } & -6.1274658257 & 10.6281448416 & 7.8676825995 \\ \text { C } & -6.1264640287 & 12.0535012994 & 7.8637209724 \\ \text { C } & 2.5098664461 & -0.0576141362 & 7.8692284914 \\ \text { C } & 2.5088617859 & 1.3685087850 & 7.9142679994 \\ \text { C } & 1.2755801388 & 2.0763894438 & 7.9387763481 \\ \text { C } & 1.2761121965 & 3.4981104733 & 8.0488184209 \\ \text { C } & 0.0356883208 & 4.2195722222 & 8.0600089262 \\ \text { C } & 0.0337889547 & 5.6509659112 & 8.0942293195\end{array}$




\begin{tabular}{|c|c|c|c|}
\hline C & -1.1977686580 & 6.3603273885 & 7.9902766201 \\
\hline C & -1.1940586356 & 7.7801294201 & 7.9612779412 \\
\hline C & -2.4255531673 & 8.4924997743 & 7.9042750021 \\
\hline C & -2.4238878887 & 9.9160523014 & 7.8743008734 \\
\hline C & -3.6580430964 & 10.6273438514 & 7.8576807453 \\
\hline C & -3.6585942737 & 12.0531624844 & 7.8579251079 \\
\hline C & 4.9778850381 & -0.0541619961 & 7.8625875048 \\
\hline C & 4.9773413506 & 1.3701089366 & 7.8969373064 \\
\hline C & 3.7410215624 & 2.0813533887 & 7.9491147404 \\
\hline C & 3.7332054356 & 3.5031078337 & 8.0782931507 \\
\hline C & 2.5014078515 & 4.1814199242 & 8.1811200367 \\
\hline C & 2.4656913976 & 5.6124924904 & 8.5428650169 \\
\hline C & 1.2408376769 & 6.3740516730 & 8.2418770722 \\
\hline C & 1.2684220473 & 7.7738986668 & 8.1268863160 \\
\hline C & 0.0422886791 & 8.4876428348 & 7.9731751872 \\
\hline C & 0.0440275165 & 9.9166433926 & 7.9163519631 \\
\hline C & -1.1887119780 & 10.6274645363 & 7.8760950443 \\
\hline C & -1.1893081745 & 12.0528863189 & 7.8606358230 \\
\hline C & 7.4455103297 & -0.0557649800 & 7.8664823524 \\
\hline C & 7.4462483277 & 1.3700599608 & 7.8763452411 \\
\hline C & 6.2126550216 & 2.0823784464 & 7.9018575329 \\
\hline C & 6.2149114932 & 3.5077354280 & 7.9508005266 \\
\hline C & 4.9871793567 & 4.2258502091 & 8.0732212610 \\
\hline C & 5.0158166441 & 5.6316380737 & 8.1626792885 \\
\hline C & 3.7982056460 & 6.3805301334 & 8.5305651537 \\
\hline C & 3.7468452465 & 7.8194133419 & 8.2141495625 \\
\hline C & 2.5189209581 & 8.4965293464 & 8.1123061326 \\
\hline C & 2.5118832963 & 9.9165474697 & 7.9747778469 \\
\hline C & 1.2771471659 & 10.6282462721 & 7.9219147552 \\
\hline C & 1.2773713834 & 12.0515749902 & 7.8843700275 \\
\hline C & 9.9122798040 & -0.0532292239 & 7.8871094576 \\
\hline C & 9.9132628567 & 1.3700424989 & 7.8819530331 \\
\hline C & 8.6807673951 & 2.0817289439 & 7.8842516366 \\
\hline C & 8.6831239722 & 3.5041524531 & 7.8932104720 \\
\hline C & 7.4492030732 & 4.2172501185 & 7.9207975707 \\
\hline C & 7.4519972951 & 5.6392956684 & 7.9305665103 \\
\hline C & 6.2201167321 & 6.3505534004 & 8.0257581487 \\
\hline
\end{tabular}




$\begin{array}{llll}\mathrm{C} & 6.2142583414 & 7.7845878086 & 8.0250377047 \\ \mathrm{C} & 4.9747800334 & 8.5026401100 & 8.0563784575 \\ \mathrm{C} & 4.9756975789 & 9.9238778166 & 7.9509130468 \\ \mathrm{C} & 3.7419331430 & 10.6314790594 & 7.9380268648 \\ \mathrm{C} & 3.7425590576 & 12.0571691212 & 7.8957290861 \\ \mathrm{C} & 12.3783777664 & -0.0522991969 & 7.8859060070 \\ \mathrm{C} & 12.3802681511 & 1.3724450425 & 7.8980754935 \\ \mathrm{C} & 11.1469477980 & 2.0834561755 & 7.8925994380 \\ \mathrm{C} & 11.1479463823 & 3.5078412868 & 7.9091851714 \\ \mathrm{C} & 9.9140844068 & 4.2177084951 & 7.8960456030 \\ \mathrm{C} & 9.9141440473 & 5.6413579828 & 7.8940677469 \\ \mathrm{C} & 8.6819564193 & 6.3542374533 & 7.9001865352 \\ \mathrm{C} & 8.6789303156 & 7.7782861375 & 7.9006369189 \\ \mathrm{C} & 7.4403387538 & 8.4910542111 & 7.9359621285 \\ \mathrm{C} & 7.4424314711 & 9.9190502334 & 7.8997207628 \\ \mathrm{C} & 6.2099625571 & 10.6317044849 & 7.9011362828 \\ \mathrm{C} & 6.2091228493 & 12.0560684810 & 7.8803222861 \\ \mathrm{C} & 3.2120694140 & 5.8898125029 & 9.8814053768 \\ \mathrm{C} & 2.7365339153 & 6.7593984708 & 10.9741710250 \\ \mathrm{H} & 3.7160124899 & 4.9996391744 & 10.2524952864 \\ \mathrm{O} & 2.9720578216 & 6.1325654403 & 12.1709345406 \\ \mathrm{O} & 2.2204806086 & 7.8640009720 & 10.9054083839 \\ \mathrm{H} & 2.6516492015 & 6.7426457414 & 12.8670581165 \\ \mathrm{Cd} & -0.1189388526 & 10.0174204487 & 11.7164464809 \\ \mathrm{Cl} & 10.4947666853 & 2.3321186082 & 4.7659953979 \\ \mathrm{Cl} & 0.1992056470 & 8.6205332189 & 4.9980233492\end{array}$

\subsubsection{Coordinates of $\mathrm{g} 6 \times 6 / 3 \mathrm{~b}-\mathrm{Hg}^{2+}, 2 \mathrm{Cl}^{-}$}

81

$\begin{array}{llll}\text { C } & 0.0420991935 & -0.0581445002 & 7.8660178236 \\ \text { C } & 0.0416583182 & 1.3655934427 & 7.8942961838 \\ \text { C } & -1.1903999694 & 2.0793516520 & 7.9104709704 \\ \text { C } & -1.1877777095 & 3.5073619958 & 7.9564559800 \\ \text { C } & -2.4260024883 & 4.2177295074 & 7.9306414397 \\ \text { C } & -2.4261257214 & 5.6416192847 & 7.9292498728 \\ \text { C } & -3.6584523811 & 6.3530342459 & 7.8956607463 \\ \text { C } & -3.6579418490 & 7.7770792230 & 7.8920720802\end{array}$




\begin{tabular}{|c|c|c|c|}
\hline C & -4.8933420847 & 8.4879473219 & 7.8803343908 \\
\hline C & -4.8924307874 & 9.9131477768 & 7.8710078623 \\
\hline C & -6.1271861504 & 10.6247775056 & 7.8666164161 \\
\hline C & -6.1259856470 & 12.0500015907 & 7.8618882492 \\
\hline C & 2.5105170572 & -0.0609667892 & 7.8756194349 \\
\hline C & 2.5095356644 & 1.3650089200 & 7.9165398410 \\
\hline C & 1.2766276849 & 2.0731903185 & 7.9324276645 \\
\hline C & 1.2770636981 & 3.4953720414 & 8.0388541990 \\
\hline C & 0.0368963588 & 4.2169373721 & 8.0423774782 \\
\hline C & 0.0343322609 & 5.6481428442 & 8.0776268331 \\
\hline C & -1.1973478705 & 6.3575236097 & 7.9780259747 \\
\hline C & -1.1936327956 & 7.7772982884 & 7.9587302810 \\
\hline C & -2.4251307846 & 8.4894091111 & 7.9117519053 \\
\hline C & -2.4234932660 & 9.9129322199 & 7.8909511868 \\
\hline C & -3.6573866587 & 10.6243005592 & 7.8710932460 \\
\hline C & -3.6579387786 & 12.0497219597 & 7.8723533289 \\
\hline C & 4.9785141850 & -0.0572845917 & 7.8792222745 \\
\hline C & 4.9778162478 & 1.3667894440 & 7.9048946309 \\
\hline C & 3.7416256875 & 2.0783099557 & 7.9514868752 \\
\hline C & 3.7334753026 & 3.5003817571 & 8.0764776443 \\
\hline C & 2.5018803145 & 4.1787614664 & 8.1769855972 \\
\hline C & 2.4637210399 & 5.6102105396 & 8.5365273425 \\
\hline C & 1.2407421278 & 6.3710537583 & 8.2285725408 \\
\hline C & 1.2686165897 & 7.7704593547 & 8.1160408580 \\
\hline C & 0.0425218764 & 8.4853055297 & 7.9709341284 \\
\hline C & 0.0440669175 & 9.9131329580 & 7.9298015488 \\
\hline C & -1.1877617016 & 10.6241367516 & 7.8964332185 \\
\hline C & -1.1888757892 & 12.0495504139 & 7.8806384012 \\
\hline C & 7.4457387074 & -0.0586333641 & 7.8811075745 \\
\hline C & 7.4464235443 & 1.3671378544 & 7.8841152832 \\
\hline C & 6.2129951693 & 2.0794756049 & 7.9054969558 \\
\hline C & 6.2149966004 & 3.5051045751 & 7.9467517677 \\
\hline C & 4.9871754069 & 4.2233764915 & 8.0707836940 \\
\hline C & 5.0149502724 & 5.6290558102 & 8.1619787217 \\
\hline C & 3.7965668929 & 6.3794060401 & 8.5261320489 \\
\hline C & 3.7467706571 & 7.8164354461 & 8.2053098510 \\
\hline C & 2.5185657157 & 8.4934349984 & 8.1056406681 \\
\hline
\end{tabular}




\begin{tabular}{|c|c|c|c|}
\hline C & 2.5119501977 & 9.9135000653 & 7.9738042870 \\
\hline C & 1.2767099821 & 10.6252196175 & 7.9326459251 \\
\hline C & 1.2773302578 & 12.0485635658 & 7.8971716266 \\
\hline C & 9.9124503249 & -0.0562826553 & 7.8931224599 \\
\hline C & 9.9136414668 & 1.3668407532 & 7.8845650233 \\
\hline C & 8.6810372623 & 2.0784547184 & 7.8870473276 \\
\hline C & 8.6836430005 & 3.5011836501 & 7.8886750338 \\
\hline C & 7.4494014298 & 4.2142011247 & 7.9135264357 \\
\hline C & 7.4519158259 & 5.6361654729 & 7.9224880837 \\
\hline C & 6.2195285585 & 6.3472101967 & 8.0197044363 \\
\hline C & 6.2142045773 & 7.7810311109 & 8.0160300652 \\
\hline C & 4.9744745341 & 8.4992661282 & 8.0462450121 \\
\hline C & 4.9757057626 & 9.9204692631 & 7.9395970807 \\
\hline C & 3.7418431333 & 10.6282952031 & 7.9315386676 \\
\hline C & 3.7428164094 & 12.0539809467 & 7.8934523238 \\
\hline C & 12.3786435034 & -0.0554958396 & 7.8785755607 \\
\hline C & 12.3811544902 & 1.3692657633 & 7.8892363014 \\
\hline C & 11.1476972574 & 2.0802458288 & 7.8867565823 \\
\hline C & 11.1489731709 & 3.5049490919 & 7.8994794149 \\
\hline C & 9.9147366138 & 4.2145590836 & 7.8887308560 \\
\hline C & 9.9144549597 & 5.6383335150 & 7.8868875848 \\
\hline C & 8.6818941961 & 6.3511454008 & 7.8939885694 \\
\hline C & 8.6792212565 & 7.7751318812 & 7.8967646385 \\
\hline C & 7.4401710172 & 8.4874448637 & 7.9285199617 \\
\hline C & 7.4425819408 & 9.9156191472 & 7.8921010930 \\
\hline C & 6.2099833543 & 10.6283506433 & 7.8901784306 \\
\hline C & 6.2094810546 & 12.0526613303 & 7.8698652460 \\
\hline C & 3.1993283123 & 5.8962874409 & 9.8767024439 \\
\hline C & 2.7006776961 & 6.7659929259 & 10.9621902185 \\
\hline $\mathrm{H}$ & 3.7074149702 & 5.0138338697 & 10.2586039065 \\
\hline O & 2.9920302489 & 6.1748350827 & 12.1662695001 \\
\hline O & 2.1224121589 & 7.8367937720 & 10.8833201346 \\
\hline $\mathrm{H}$ & 2.6482674162 & 6.7791000841 & 12.8566057833 \\
\hline H & -0.0862904337 & 10.4370519107 & 11.9499721364 \\
\hline $\mathrm{Cl}$ & 10.4887463025 & 2.2713748406 & 4.7693239257 \\
\hline & 0.2901007333 & 8.4026459072 & 4.9764899102 \\
\hline
\end{tabular}




\subsection{Coordinates of $\mathrm{g} 6 \times 6 / 3 \mathrm{c}-\mathrm{M}^{2+}, \mathrm{NaCl}, \mathrm{Cl}^{-}$}

\subsubsection{Coordinates of $\mathrm{g} 6 \times 6 / 3 \mathrm{c}-\mathrm{Pb}^{2+}, \mathrm{NaCl}, \mathrm{Cl}^{-}$}

81

$\begin{array}{llll}\text { C } & 0.0478888852 & -0.0128900977 & 7.8333339543 \\ \text { C } & 0.0471939640 & 1.4114823683 & 7.8534300587 \\ \text { C } & -1.1830440162 & 2.1259884548 & 7.8640411818 \\ \text { C } & -1.1807031168 & 3.5532919992 & 7.9116473556 \\ \text { C } & -2.4181538005 & 4.2651040501 & 7.8790312846 \\ \text { C } & -2.4205209069 & 5.6872985227 & 7.8838570601 \\ \text { C } & -3.6533358248 & 6.4004512241 & 7.8579947497 \\ \text { C } & -3.6541337466 & 7.8246076144 & 7.8525274658 \\ \text { C } & -4.8870327572 & 8.5348911888 & 7.8488167834 \\ \text { C } & -4.8859907361 & 9.9597233531 & 7.8339079271 \\ \text { C } & -6.1207078880 & 10.6710120742 & 7.8389086311 \\ \text { C } & -6.1205585899 & 12.0952403331 & 7.8273212461 \\ \text { C } & 2.5158188115 & -0.0150852370 & 7.8280072580 \\ \text { C } & 2.5143629053 & 1.4110995855 & 7.8683075477 \\ \text { C } & 1.2811899134 & 2.1198417842 & 7.8887047555 \\ \text { C } & 1.2792727003 & 3.5406377027 & 7.9932974261 \\ \text { C } & 0.0424503183 & 4.2601262573 & 7.9986146728 \\ \text { C } & 1.2460301880 & 6.4125300025 & 8.1630379041 \\ \text { C } & 0.0398497519 & 5.6914330322 & 8.0284698180 \\ \text { C } & 3.7370472836 & 3.5453995887 & 8.0313206864 \\ \text { C } & -1.1904641906 & 6.4017687154 & 7.9306437423 \\ \text { C } & -1.1883100727 & 7.8238548188 & 7.9064696453 \\ \text { C } & -2.4223276697 & 8.5371543940 & 7.8583517482 \\ \text { C } & -2.4210686595 & 9.9579271527 & 7.8321599420 \\ \text { C } & -3.6544743438 & 10.6699858337 & 7.8197270480 \\ \text { C } & -3.6548229056 & 12.0950063529 & 7.8167408977 \\ \text { C } & 4.9819590828 & -0.0111463519 & 7.8178383262 \\ \text { C } & 4.9810138141 & 1.4121131955 & 7.8536933469 \\ \text { C } & 3.7460277995 & 2.1235499162 & 7.9040546259 \\ \text { C } & 7.8153845607 & 8.0527824706\end{array}$




\begin{tabular}{|c|c|c|c|}
\hline C & 0.0452196906 & 8.5323488121 & 7.9246775654 \\
\hline C & 0.0465627469 & 9.9572150749 & 7.8656319034 \\
\hline C & -1.1857707160 & 10.6690957388 & 7.8287237692 \\
\hline C & -1.1855777643 & 12.0956449681 & 7.8138259508 \\
\hline C & 7.4487385134 & -0.0129441211 & 7.8180079116 \\
\hline C & 7.4490126928 & 1.4137810252 & 7.8278051351 \\
\hline C & 6.2167406628 & 2.1263332882 & 7.8599268906 \\
\hline C & 6.2188599719 & 3.5516804561 & 7.9166316187 \\
\hline C & 4.9898953233 & 4.2699951967 & 8.0395879499 \\
\hline C & 5.0166194989 & 5.6750561963 & 8.1409925475 \\
\hline C & 3.7876613004 & 6.4208981997 & 8.4801186220 \\
\hline C & 3.7507102391 & 7.8606742745 & 8.1712219895 \\
\hline C & 2.5237104793 & 8.5379706267 & 8.0527700508 \\
\hline C & 2.5175509072 & 9.9602455174 & 7.9227871460 \\
\hline C & 1.2824763939 & 10.6710969911 & 7.8661261745 \\
\hline C & 1.2820993701 & 12.0945321252 & 7.8309834887 \\
\hline C & 9.9191943648 & -0.0111723116 & 7.8302386259 \\
\hline C & 9.9205040971 & 1.4148017235 & 7.8224406668 \\
\hline C & 8.6851819342 & 2.1267302195 & 7.8276993290 \\
\hline C & 8.6873301477 & 3.5487243056 & 7.8423276677 \\
\hline C & 7.4520736384 & 4.2620780715 & 7.8875619636 \\
\hline C & 7.4559475268 & 5.6846165285 & 7.9108534821 \\
\hline C & 6.2242184184 & 6.3950261450 & 8.0158989911 \\
\hline C & 6.2219026663 & 7.8278171280 & 8.0138946624 \\
\hline C & 4.9810472611 & 8.5445804154 & 8.0340344044 \\
\hline C & 4.9825535128 & 9.9656807709 & 7.9212389962 \\
\hline C & 3.7489073313 & 10.6735535827 & 7.8951958850 \\
\hline C & 3.7493023053 & 12.1003225401 & 7.8461914619 \\
\hline C & 12.3859384267 & -0.0094742558 & 7.8353630961 \\
\hline C & 12.3880634803 & 1.4150718557 & 7.8361217679 \\
\hline C & 11.1527279298 & 2.1265962790 & 7.8312437442 \\
\hline C & 11.1546883912 & 3.5522023535 & 7.8417961937 \\
\hline C & 9.9202326534 & 4.2628161211 & 7.8382438782 \\
\hline C & 9.9201075857 & 5.6875284520 & 7.8491486116 \\
\hline C & 8.6870042354 & 6.3997684349 & 7.8731284287 \\
\hline C & 8.6857405121 & 7.8226938018 & 7.8799728683 \\
\hline C & 7.4467013601 & 8.5338687143 & 7.92136162 \\
\hline
\end{tabular}




\section{Supporting information}

$\begin{array}{llll}\mathrm{C} & 7.4496662727 & 9.9619728310 & 7.8773489867 \\ \mathrm{C} & 6.2179394645 & 10.6739474183 & 7.8722574122 \\ \mathrm{C} & 6.2174740070 & 12.0983507162 & 7.8399883423 \\ \mathrm{C} & 3.1842184134 & 5.9270043717 & 9.8535248607 \\ \mathrm{C} & 2.6410652054 & 6.7007870075 & 10.9463926271 \\ \mathrm{H} & 3.6930421898 & 5.0375411274 & 10.2184830639 \\ \mathrm{O} & 2.7825141092 & 6.1967469135 & 12.1470337857 \\ \mathrm{O} & 2.0090102872 & 7.8255667289 & 10.8306259737 \\ \mathrm{~Pb} & 1.5882271711 & 7.8949724177 & 13.0934470641 \\ \mathrm{Cl} & 0.0181328672 & 5.1545038022 & 4.6150159734 \\ \mathrm{Cl} & -3.0601323327 & 8.9750018501 & 4.3867641793 \\ \mathrm{Na} & -1.5090025460 & 7.0448969430 & 3.9693147257\end{array}$

\subsubsection{Coordinates of $\mathrm{g} 6 \times 6 / 3 \mathrm{c}-\mathrm{Cd}^{2+}, \mathrm{NaCl}, \mathrm{Cl}^{-}$}

81

$\begin{array}{llll}\text { C } & 0.0048005850 & -0.0276543313 & 7.8204065996 \\ \text { C } & 0.0046034569 & 1.3965625571 & 7.8356114262 \\ \text { C } & -1.2266767512 & 2.1111267764 & 7.8489711406 \\ \text { C } & -1.2244046940 & 3.5393474524 & 7.8996097805 \\ \text { C } & -2.4623640182 & 4.2511868487 & 7.8714194722 \\ \text { C } & -2.4640089508 & 5.6742498687 & 7.8844425130 \\ \text { C } & -3.6965701169 & 6.3867351051 & 7.8563882897 \\ \text { C } & -3.6968074689 & 7.8095638510 & 7.8581596425 \\ \text { C } & -4.9307744042 & 8.5197147082 & 7.8467971434 \\ \text { C } & -4.9300939550 & 9.9442419295 & 7.8340504756 \\ \text { C } & -6.1646277218 & 10.6556268366 & 7.8314121759 \\ \text { C } & -6.1635853485 & 12.0805394265 & 7.8165410162 \\ \text { C } & 2.4721858144 & -0.0293190376 & 7.8182420698 \\ \text { C } & 2.4710658095 & 1.3967990491 & 7.8491793070 \\ \text { C } & 1.2386139108 & 2.1056811098 & 7.8653209023 \\ \text { C } & 1.2386709608 & 3.5274869229 & 7.9718720025 \\ \text { C } & 0.0003410052 & 4.2477281337 & 7.9915509585 \\ \text { C } & -0.0028355927 & 5.6779778956 & 8.0408431261 \\ \text { C } & -1.2336216050 & 6.3883972820 & 7.9468815182 \\ \text { C } & -1.2316776930 & 7.8102757859 & 7.9410350281 \\ \text { C } & -2.4647243584 & 8.5221488125 & 7.8806838607 \\ \text { C } & -2.4637371868 & 9.9440634178 & 7.8580341545\end{array}$




\begin{tabular}{|c|c|c|c|}
\hline C & -3.6970979067 & 10.6556741142 & 7.8303451161 \\
\hline C & -3.6973510513 & 12.0803323434 & 7.8206346203 \\
\hline C & 4.9389410630 & -0.0255398910 & 7.8238315305 \\
\hline C & 4.9377846319 & 1.3986934791 & 7.8461523446 \\
\hline C & 3.7024228222 & 2.1109779457 & 7.8879644170 \\
\hline C & 3.6936955084 & 3.5322305440 & 8.0169355699 \\
\hline C & 2.4629850128 & 4.2115724765 & 8.1079536991 \\
\hline C & 2.4232491450 & 5.6334118426 & 8.4928725327 \\
\hline C & 1.2052764096 & 6.3978905636 & 8.1891271170 \\
\hline C & 1.2293239232 & 7.8041072652 & 8.1125858503 \\
\hline C & 0.0031971605 & 8.5183961949 & 7.9834503900 \\
\hline C & 0.0039566912 & 9.9435660681 & 7.9179702641 \\
\hline C & -1.2286879758 & 10.6551453205 & 7.8649825991 \\
\hline C & -1.2288574142 & 12.0812981525 & 7.8343180443 \\
\hline C & 7.4062377631 & -0.0272100072 & 7.8318199344 \\
\hline C & 7.4058722199 & 1.3992655170 & 7.8251797665 \\
\hline C & 6.1730992685 & 2.1123870281 & 7.8495416400 \\
\hline C & 6.1745108899 & 3.5382974777 & 7.8999398378 \\
\hline C & 4.9468625059 & 4.2558829152 & 8.0275462720 \\
\hline C & 4.9750894466 & 5.6609333744 & 8.1339257449 \\
\hline C & 3.7597407808 & 6.4078103608 & 8.5124761857 \\
\hline C & 3.7093790672 & 7.8452420623 & 8.2001022794 \\
\hline C & 2.4801357055 & 8.5246269957 & 8.1060384977 \\
\hline C & 2.4743621403 & 9.9439896506 & 7.9663729556 \\
\hline C & 1.2392209927 & 10.6559628363 & 7.9095976917 \\
\hline C & 1.2388757509 & 12.0798570011 & 7.8540443789 \\
\hline C & 9.8751985696 & -0.0262354359 & 7.8416172569 \\
\hline C & 9.8762930680 & 1.3993532224 & 7.8223652621 \\
\hline C & 8.6420145803 & 2.1115476640 & 7.8222085860 \\
\hline C & 8.6436951488 & 3.5344694162 & 7.8302536916 \\
\hline C & 7.4090058613 & 4.2476695776 & 7.8686177087 \\
\hline C & 7.4126609556 & 5.6693597256 & 7.8884518666 \\
\hline C & 6.1808454526 & 6.3792008068 & 7.9956838735 \\
\hline C & 6.1768515286 & 7.8121592554 & 7.9999383053 \\
\hline C & 4.9356038288 & 8.5289734309 & 8.0369653042 \\
\hline C & 4.9382060413 & 9.9505415651 & 7.9292259243 \\
\hline C & 3.7051863929 & 10.6586341761 & 7.9175685214 \\
\hline
\end{tabular}




$\begin{array}{lccc}\mathrm{C} & 3.7059241658 & 12.0840821636 & 7.8577416572 \\ \mathrm{C} & 12.3420295480 & -0.0253837768 & 7.8337813763 \\ \mathrm{C} & 12.3443297387 & 1.4001253992 & 7.8281217590 \\ \mathrm{C} & 11.1094837455 & 2.1120844390 & 7.8258868348 \\ \mathrm{C} & 11.1103854512 & 3.5371757432 & 7.8340148018 \\ \mathrm{C} & 9.8760185997 & 4.2482353270 & 7.8288749850 \\ \mathrm{C} & 9.8764280013 & 5.6728757204 & 7.8390487673 \\ \mathrm{C} & 8.6434845604 & 6.3843674910 & 7.8562974079 \\ \mathrm{C} & 8.6416328527 & 7.8074191612 & 7.8666645220 \\ \mathrm{C} & 7.4025763255 & 8.5187402464 & 7.9072271762 \\ \mathrm{C} & 7.4055056176 & 9.9464737463 & 7.8684125540 \\ \mathrm{C} & 6.1729542892 & 10.6585228712 & 7.8701017842 \\ \mathrm{C} & 6.1731721198 & 12.0826113086 & 7.8352413844 \\ \mathrm{C} & 3.1508603113 & 5.8934707645 & 9.8396545991 \\ \mathrm{C} & 2.6190043628 & 6.6900347327 & 10.9911324401 \\ \mathrm{H} & 3.6508808868 & 4.9965763829 & 10.1985211610 \\ \mathrm{O} & 2.6834971078 & 6.0651789635 & 12.1091803378 \\ \mathrm{O} & 2.1512597885 & 7.8650152322 & 10.8642026854 \\ \mathrm{Cd} & 1.7340120898 & 7.8875499221 & 13.2447258756 \\ \mathrm{Cl} & 1.5434857700 & 6.2192870310 & 4.9476116659 \\ \mathrm{Cl} & 12.0452556661 & 8.5738960888 & 4.4382365863 \\ \mathrm{Na} & -0.5654676495 & 7.3724826042 & 4.1495414528\end{array}$

\subsubsection{Coordinates of $\mathrm{g} 6 \times 6 / 3 \mathrm{c}-\mathrm{Hg}^{2+}, \mathrm{NaCl}, \mathrm{Cl}$}

81

$\begin{array}{llll}\text { C } & 0.0583100986 & -0.0106691222 & 7.9017909739 \\ \text { C } & 0.0555473379 & 1.4134269345 & 7.9159717680 \\ \text { C } & -1.1748039126 & 2.1270010415 & 7.9338274250 \\ \text { C } & -1.1747256432 & 3.5500687570 & 7.9686396013 \\ \text { C } & -2.4085386859 & 4.2648126847 & 7.9545320840 \\ \text { C } & -2.4112375826 & 5.6920229797 & 7.9470030197 \\ \text { C } & -3.6409210898 & 6.4033291915 & 7.9291097356 \\ \text { C } & -3.6430715260 & 7.8287178764 & 7.9204629529 \\ \text { C } & -4.8773472851 & 8.5404925048 & 7.9115506362 \\ \text { C } & -4.8775698631 & 9.9625899213 & 7.8979671809 \\ \text { C } & -6.1116670333 & 10.6769258941 & 7.8964638907 \\ \text { C } & -6.1121515451 & 12.1006910668 & 7.8868885766\end{array}$




\begin{tabular}{|c|c|c|c|}
\hline C & 2.5257163316 & -0.0011723183 & 7.8745034541 \\
\hline C & 2.5256923086 & 1.4143883649 & 7.8703031767 \\
\hline C & 1.2867023109 & 2.1275426978 & 7.8949722688 \\
\hline C & 1.2695325551 & 3.5390525021 & 7.9105581755 \\
\hline C & 0.0609290861 & 4.2567882331 & 7.9852950242 \\
\hline C & 0.0550192795 & 5.7013616961 & 8.0013660428 \\
\hline C & -1.1798551960 & 6.4072941249 & 7.9611362576 \\
\hline C & -1.1786073678 & 7.8283860174 & 7.9520580962 \\
\hline C & -2.4141003071 & 8.5405188976 & 7.9242804850 \\
\hline C & -2.4113554105 & 9.9635129542 & 7.9043001323 \\
\hline C & -3.6442197085 & 10.6769793111 & 7.8903469518 \\
\hline C & -3.6404836951 & 12.1004453993 & 7.8807991614 \\
\hline C & 4.9957878526 & -0.0107529599 & 7.8875397240 \\
\hline C & 5.0011941819 & 1.4129837785 & 7.9034963797 \\
\hline C & 3.7673894476 & 2.1314060422 & 7.8955358570 \\
\hline C & 3.7842818436 & 3.5388616384 & 7.9192503426 \\
\hline C & 2.5260890836 & 4.2820911636 & 7.7427149672 \\
\hline C & 2.4931147183 & 5.6518844171 & 8.3391786269 \\
\hline C & 1.2564329617 & 6.4193604330 & 8.0912997622 \\
\hline C & 1.2839790379 & 7.8247268062 & 8.0507208532 \\
\hline C & 0.0543963519 & 8.5382572194 & 7.9683736684 \\
\hline C & 0.0585917996 & 9.9629423276 & 7.9267645751 \\
\hline C & -1.1752935409 & 10.6740165672 & 7.9010412540 \\
\hline C & -1.1724145115 & 12.0967606474 & 7.8876912644 \\
\hline C & 7.4641781782 & -0.0135370831 & 7.8856033777 \\
\hline C & 7.4683400729 & 1.4105091226 & 7.8931013388 \\
\hline C & 6.2336789728 & 2.1236705211 & 7.9108669785 \\
\hline C & 6.2395839536 & 3.5462701635 & 7.9496709423 \\
\hline C & 5.0051791545 & 4.2562670899 & 7.9933896928 \\
\hline C & 5.0228119816 & 5.6837665961 & 8.0700617088 \\
\hline C & 3.8028487977 & 6.4211475108 & 8.3358130260 \\
\hline C & 3.7672488532 & 7.8568377324 & 8.1181628203 \\
\hline C & 2.5337682862 & 8.5437522726 & 8.0492418924 \\
\hline C & 2.5296190392 & 9.9591776357 & 7.9559254754 \\
\hline C & 1.2930413604 & 10.6721389217 & 7.9192635409 \\
\hline C & 1.2946734264 & 12.0945766289 & 7.8919621520 \\
\hline C & 9.9306472257 & -0.0110126157 & 7.8936106141 \\
\hline
\end{tabular}




$\begin{array}{lccc}\mathrm{C} & 9.9321041248 & 1.4113924463 & 7.8979870618 \\ \mathrm{C} & 8.6988278892 & 2.1237496058 & 7.9009137704 \\ \mathrm{C} & 8.7012940317 & 3.5494526889 & 7.9168166793 \\ \mathrm{C} & 7.4704385915 & 4.2606335556 & 7.9393788927 \\ \mathrm{C} & 7.4723930848 & 5.6848375269 & 7.9415683404 \\ \mathrm{C} & 6.2380003735 & 6.3961101576 & 7.9950607612 \\ \mathrm{C} & 6.2313093491 & 7.8281684161 & 7.9912333353 \\ \mathrm{C} & 4.9937544546 & 8.5464845121 & 8.0032734104 \\ \mathrm{C} & 4.9940500600 & 9.9684940512 & 7.9373571360 \\ \mathrm{C} & 3.7621357082 & 10.6744005294 & 7.9302512815 \\ \mathrm{C} & 3.7614573461 & 12.0982894633 & 7.9037370456 \\ \mathrm{C} & 12.3951936345 & -0.0101457809 & 7.9048114032 \\ \mathrm{C} & 12.3944172966 & 1.4137498003 & 7.9153396203 \\ \mathrm{C} & 11.1649622371 & 2.1255508685 & 7.9147552835 \\ \mathrm{C} & 11.1663775883 & 3.5523520578 & 7.9285965327 \\ \mathrm{C} & 9.9328649243 & 4.2652153823 & 7.9199115033 \\ \mathrm{C} & 9.9321992005 & 5.6881748983 & 7.9221252501 \\ \mathrm{C} & 8.6992128683 & 6.4002678275 & 7.9238722599 \\ \mathrm{Cl} & 10.4358873886 & 8.2291202439 & 4.3405160110 \\ \mathrm{C} & 8.6957665838 & 7.8259429177 & 7.9238390661 \\ \mathrm{C} & 7.4602428341 & 8.5372917157 & 7.9403822363 \\ \mathrm{C} & 7.4607201568 & 9.9652385540 & 7.9164447444 \\ \mathrm{C} & 6.2284414040 & 10.6775291889 & 7.9135720984 \\ \mathrm{C} & 6.2272418720 & 12.0989712872 & 7.9053856048 \\ \mathrm{C} & 3.1179880041 & 5.8754217661 & 9.6962538588 \\ \mathrm{C} & 2.5461820289 & 6.8169474746 & 10.7813024504 \\ \mathrm{C} & 3.6629359943 & 5.0254331152 & 10.1077035738 \\ \mathrm{C} & 1.6356613293 & 6.1611575258 & 11.3739159864 \\ \mathrm{C} & 3.0094293400 & 7.9527043957 & 10.9629993920 \\ \mathrm{C} & 0.8495285465 & 8.1229692254 & 13.4518697313 \\ \mathrm{C} & 2.5577401131 & 4.6600289779 & 5.7241758826 \\ \mathrm{C} & & & \end{array}$

\subsection{Coordinates of $\mathrm{g} 6 \times 6 / 3 \mathrm{~d}-\mathrm{M}^{2+}, 2 \mathrm{Cl}^{-}$}

\subsubsection{Coordinates of $\mathrm{g} 6 \times 6 / 3 \mathrm{~d}-\mathrm{Pb}^{2+}, 2 \mathrm{C}^{\mathrm{l}}$}




\begin{tabular}{|c|c|c|c|}
\hline C & -1.3796913884 & -0.7257182407 & 7.7498372392 \\
\hline $\mathrm{C}$ & -1.3794284431 & 0.6990108243 & 7.7253928117 \\
\hline & -2.6093910220 & 1.4126163358 & 7.6990936972 \\
\hline & -2.6048488763 & 2.8423969392 & 7.6786811886 \\
\hline & -3.8455583586 & 3.5535580015 & 7.6935057104 \\
\hline & -3.8487069511 & 4.9754046913 & 7.7202149056 \\
\hline & -5.0828604871 & 5.6874784300 & 7.7441589478 \\
\hline & -5.0837765214 & 7.1117381185 & 7.7776227194 \\
\hline & -6.3183960760 & 7.8205299380 & 7.7946589800 \\
\hline & -6.3155802220 & 9.2465238071 & 7.8101346434 \\
\hline & -7.5517205551 & 9.9572095598 & 7.7962989159 \\
\hline & -7.5491751759 & 11.3809793768 & 7.7765741560 \\
\hline & 1.0879922917 & -0.7304211628 & 7.7810723366 \\
\hline & 1.0875362436 & 0.6966466824 & 7.7611058746 \\
\hline & -0.1441014882 & 1.4077086624 & 7.7288789050 \\
\hline & -0.1402450675 & 2.8325218074 & 7.6842892369 \\
\hline & -1.3798602687 & 3.5496877679 & 7.6615974806 \\
\hline & -1.3813586066 & 4.9819107429 & 7.6821399981 \\
\hline & -2.6171410854 & 5.6913209106 & 7.7276375413 \\
\hline & -2.6174503306 & 7.1131414822 & 7.7664606841 \\
\hline & -3.8523837788 & 7.8260614993 & 7.7892374037 \\
\hline & -3.8503571949 & 9.2457061548 & 7.8063766829 \\
\hline & -5.0850287469 & 9.9566121461 & 7.8141021784 \\
\hline & -5.0834384743 & 11.3812974679 & 7.8018551863 \\
\hline & 3.5538308864 & -0.7253159469 & 7.7883645764 \\
\hline & 3.5545252139 & 0.6957668240 & 7.7694523899 \\
\hline & 2.3193887092 & 1.4082787160 & 7.7725455477 \\
\hline & 2.3121748335 & 2.8324430176 & 7.7587777719 \\
\hline & 1.0896851514 & 3.5217884708 & 7.6857134991 \\
\hline & 1.1137177054 & 4.9887740172 & 7.4830290228 \\
\hline & -0.1693937368 & 5.7027769164 & 7.6836205563 \\
\hline & -0.1564458464 & 7.1067931851 & 7.7630033187 \\
\hline & -1.3857394314 & 7.8249564886 & 7.7816832900 \\
\hline & -1.3844464952 & 9.2506336533 & 7.7872159341 \\
\hline & -2.6147506710 & 9.9589981732 & 7.7992373653 \\
\hline & -2.6139425468 & 11.3835700055 & 7.78520746 \\
\hline & 6.0187855573 & -0.7253906533 & 7.7568986810 \\
\hline
\end{tabular}




\begin{tabular}{|c|c|c|c|}
\hline C & 6.0209498091 & 0.7000954232 & 7.7328995324 \\
\hline C & 4.7900313898 & 1.4105186511 & 7.7330508417 \\
\hline C & 4.7926949887 & 2.8355248235 & 7.7168638924 \\
\hline C & 3.5641588224 & 3.5550903537 & 7.7520330691 \\
\hline C & 3.5820824525 & 4.9517383343 & 7.8492826198 \\
\hline C & 2.2973637760 & 5.6668835806 & 8.1705435871 \\
\hline C & 2.3181143452 & 7.1467836524 & 7.8523043879 \\
\hline C & 1.0964542674 & 7.8311097528 & 7.7707038526 \\
\hline C & 1.0876460151 & 9.2561404887 & 7.7427499832 \\
\hline C & -0.1486374652 & 9.9643520115 & 7.7538736014 \\
\hline C & -0.1474138878 & 11.3853476070 & 7.7447069440 \\
\hline C & 8.4906425596 & -0.7217275872 & 7.7265962354 \\
\hline C & 8.4931098902 & 0.7034184233 & 7.7127793749 \\
\hline C & 7.2573357485 & 1.4137406112 & 7.7145804474 \\
\hline C & 7.2600576087 & 2.8345853306 & 7.7130054984 \\
\hline C & 6.0235299600 & 3.5472652747 & 7.7223711730 \\
\hline C & 6.0241417688 & 4.9694219573 & 7.7451776898 \\
\hline C & 4.7877141758 & 5.6754096035 & 7.8059731103 \\
\hline C & 4.7872400558 & 7.1101245979 & 7.8303425289 \\
\hline C & 3.5476637263 & 7.8317455667 & 7.8201008782 \\
\hline C & 3.5524043033 & 9.2553666788 & 7.7647417559 \\
\hline C & 2.3198888798 & 9.9657714077 & 7.7447698155 \\
\hline C & 2.3208785447 & 11.3918508050 & 7.7319986422 \\
\hline C & 10.9583699063 & -0.7198047613 & 7.7273013828 \\
\hline C & 10.9608437088 & 0.7034732580 & 7.7074444392 \\
\hline C & 9.7246641424 & 1.4141456393 & 7.7015019184 \\
\hline C & 9.7270765544 & 2.8397913757 & 7.6989311074 \\
\hline $\mathrm{C}$ & 8.4925665121 & 3.5493101936 & 7.7118283512 \\
\hline C & 8.4911929533 & 4.9742584472 & 7.7323817225 \\
\hline C & 7.2561326348 & 5.6852169940 & 7.7523476098 \\
\hline $\mathrm{C}$ & 7.2542778040 & 7.1070525103 & 7.7870584986 \\
\hline C & 6.0121464325 & 7.8173436502 & 7.8060462331 \\
\hline C & 6.0178668107 & 9.2482509535 & 7.7923321281 \\
\hline C & 4.7887129619 & 9.9627936279 & 7.7651869039 \\
\hline C & 4.7896772680 & 11.3873753422 & 7.7452078150 \\
\hline C & 2.0681784635 & 5.6873368885 & 9.7023019469 \\
\hline C & 2.8678066227 & 5.2616017476 & 10.7110319806 \\
\hline
\end{tabular}




$\begin{array}{llll}\mathrm{H} & 1.1871714082 & 6.2550485808 & 10.0051515036 \\ \mathrm{C} & 2.5871002122 & 5.6626805982 & 12.1290877108 \\ \mathrm{O} & 3.9894461268 & 4.5222814719 & 10.5919525755 \\ \mathrm{C} & 2.4761273615 & 4.6686801092 & 13.1408156235 \\ \mathrm{C} & 2.3941835410 & 5.0418175080 & 14.4946314874 \\ \mathrm{C} & 2.4075372127 & 6.3900284003 & 14.8522866381 \\ \mathrm{C} & 2.4741016443 & 7.3732348508 & 13.8576796203 \\ \mathrm{C} & 2.5684229326 & 7.0147294037 & 12.5116976895 \\ \mathrm{H} & 2.6499888274 & 7.7805017722 & 11.7384478411 \\ \mathrm{H} & 2.3503235445 & 6.6761184511 & 15.9026757199 \\ \mathrm{H} & 2.4755037452 & 8.4277441827 & 14.1360266059 \\ \mathrm{H} & 2.3430396095 & 3.6224986205 & 12.8514360591 \\ \mathrm{H} & 2.2873220205 & 4.2752746721 & 15.2634810521 \\ \mathrm{~Pb} & 5.1320339239 & 4.3315195643 & 12.4449324469 \\ \mathrm{Cl} & 1.4623131742 & 5.1947180170 & 5.5575886922 \\ \mathrm{Cl} & -3.6922795402 & 9.7918087722 & 4.5991323967\end{array}$

\subsubsection{Coordinates of $\mathrm{g} 6 \times 6 / 3 \mathrm{~d}-\mathrm{Cd}^{2+}, 2 \mathrm{Cl}^{-}$}

90

$\begin{array}{llll}\text { C } & -0.3848979852 & 0.0407908766 & 7.6820184930 \\ \text { C } & -0.3875142540 & 1.4643448239 & 7.6817190398 \\ \text { C } & -1.6209829753 & 2.1759441237 & 7.6735377532 \\ \text { C } & -1.6223155215 & 3.5998310319 & 7.6838837703 \\ \text { C } & -2.8541730028 & 4.3125840896 & 7.6684392771 \\ \text { C } & -2.8513777956 & 5.7378370323 & 7.6746824206 \\ \text { C } & -4.0860248150 & 6.4506225419 & 7.6616624197 \\ \text { C } & -4.0851842963 & 7.8748019624 & 7.6658578340 \\ \text { C } & -5.3204893959 & 8.5870353035 & 7.6766138959 \\ \text { C } & -5.3191287052 & 10.0127917168 & 7.6735257907 \\ \text { C } & -6.5507511097 & 10.7255464186 & 7.6891324578 \\ \text { C } & -6.5530512074 & 12.1494166107 & 7.6774135139 \\ \text { C } & 2.0827560271 & 0.0428268155 & 7.6794934718 \\ \text { C } & 2.0804904632 & 1.4664189928 & 7.7148300381 \\ \text { C } & 0.8461423969 & 2.1760141212 & 7.7117274291 \\ \text { C } & 0.8418524348 & 3.5983980205 & 7.7610873469 \\ \text { C } & -0.3885323538 & 4.3124217988 & 7.7409369527 \\ \text { C } & -0.3861633896 & 5.7360549815 & 7.7829781349\end{array}$




\begin{tabular}{|c|c|c|c|}
\hline C & -1.6187058856 & 6.4504131341 & 7.7211180947 \\
\hline C & -1.6186814554 & 7.8749254637 & 7.7104806225 \\
\hline C & -2.8520514331 & 8.5878653006 & 7.6758867786 \\
\hline C & -2.8521205381 & 10.0124443544 & 7.6729886562 \\
\hline C & -4.0848663881 & 10.7259177789 & 7.6648598932 \\
\hline C & -4.0857550282 & 12.1506066098 & 7.6717501619 \\
\hline C & 4.5502118789 & 0.0432362595 & 7.6770620128 \\
\hline C & 4.5522935164 & 1.4657991186 & 7.7136346396 \\
\hline C & 3.3165515953 & 2.1820372012 & 7.7603070577 \\
\hline C & 3.3167124946 & 3.5966175468 & 7.8635569289 \\
\hline C & 2.0729877490 & 4.3087923599 & 7.8747134847 \\
\hline C & 2.0523440854 & 5.7132480730 & 8.0182924995 \\
\hline C & 0.8491884864 & 6.4400132925 & 7.9167461171 \\
\hline C & 0.8469981674 & 7.8880196534 & 7.9068549002 \\
\hline C & -0.3842638684 & 8.5910050982 & 7.7704926272 \\
\hline C & -0.3870457244 & 10.0145358440 & 7.7301948286 \\
\hline C & -1.6206744616 & 10.7258147128 & 7.6835452991 \\
\hline C & -1.6187982377 & 12.1490263894 & 7.6708651122 \\
\hline C & 7.0160530600 & 0.0417436628 & 7.6727120032 \\
\hline C & 7.0188193003 & 1.4652029247 & 7.6764440906 \\
\hline C & 5.7860424512 & 2.1769300171 & 7.7083575227 \\
\hline C & 5.7898071177 & 3.5996185687 & 7.7609145916 \\
\hline C & 4.5600065014 & 4.3120629545 & 7.8850118646 \\
\hline C & 4.5825437649 & 5.7151565426 & 8.0432373979 \\
\hline C & 3.3148251956 & 6.3952113556 & 8.3775408500 \\
\hline C & 3.3102295788 & 7.9365970837 & 8.3339868468 \\
\hline C & 2.0527770967 & 8.6153570982 & 7.9811628592 \\
\hline C & 2.0724617338 & 10.0185642827 & 7.8378795278 \\
\hline C & 0.8405488567 & 10.7289714092 & 7.7403934628 \\
\hline C & 0.8464342867 & 12.1515528711 & 7.6975059586 \\
\hline C & 9.4820671283 & 0.0411471564 & 7.7041788515 \\
\hline C & 9.4837547645 & 1.4615754954 & 7.6720355050 \\
\hline C & 8.2522140135 & 2.1762207633 & 7.6718528621 \\
\hline C & 8.2533262358 & 3.5990789617 & 7.6808516509 \\
\hline C & 7.0197653606 & 4.3121109085 & 7.7345366389 \\
\hline C & 7.0152096237 & 5.7358800643 & 7.7689988730 \\
\hline C & 5.7836610602 & 6.4391238688 & 7.9129707282 \\
\hline
\end{tabular}




\begin{tabular}{|c|c|c|c|}
\hline C & 5.7830023437 & 7.8832664884 & 7.9174720771 \\
\hline C & 4.5789402116 & 8.6103263084 & 8.0190156981 \\
\hline C & 4.5584207940 & 10.0154857370 & 7.8636412537 \\
\hline C & 3.3157959589 & 10.7314893716 & 7.8330558480 \\
\hline C & 3.3155248718 & 12.1455240787 & 7.7438387913 \\
\hline C & 11.9530246873 & 0.0408044356 & 7.7090413053 \\
\hline C & 11.9508811490 & 1.4622104225 & 7.6748207021 \\
\hline C & 10.7178407363 & 2.1750503180 & 7.6573967759 \\
\hline C & 10.7174403994 & 3.5997465565 & 7.6560336777 \\
\hline C & 9.4852281547 & 4.3117744737 & 7.6654141021 \\
\hline C & 9.4834837794 & 5.7373861302 & 7.6702650319 \\
\hline C & 8.2495701049 & 6.4497037434 & 7.7113299608 \\
\hline C & 8.2500501808 & 7.8750050064 & 7.7160824786 \\
\hline C & 7.0163095169 & 8.5891803100 & 7.7781402468 \\
\hline C & 7.0184611631 & 10.0123776765 & 7.7409376592 \\
\hline C & 5.7889636044 & 10.7268560267 & 7.7581601284 \\
\hline C & 5.7838849060 & 12.1502774035 & 7.7088473867 \\
\hline C & 3.2054867455 & 7.1778257323 & 9.7047749900 \\
\hline C & 4.2219247591 & 7.2386428180 & 10.7943552327 \\
\hline $\mathrm{H}$ & 2.1792749747 & 7.2028107096 & 10.0695461459 \\
\hline C & 3.7618327902 & 7.8413696720 & 12.0888356478 \\
\hline $\mathrm{O}$ & 5.3736948402 & 6.8153344945 & 10.6774912909 \\
\hline C & 4.5192824917 & 7.5588986409 & 13.2383520621 \\
\hline C & 4.1739578162 & 8.1110788073 & 14.4675695077 \\
\hline C & 3.0768545850 & 8.9730727740 & 14.5651221852 \\
\hline C & 2.3276429857 & 9.2763855028 & 13.4257008096 \\
\hline C & 2.6589024621 & 8.7060145423 & 12.1954860434 \\
\hline $\mathrm{H}$ & 2.0678783588 & 8.9667600721 & 11.3161628142 \\
\hline $\mathrm{H}$ & 2.8051393094 & 9.4057864710 & 15.5291672014 \\
\hline $\mathrm{H}$ & 1.4826005323 & 9.9616873172 & 13.4950583155 \\
\hline $\mathrm{H}$ & 5.3800626937 & 6.8971143124 & 13.1388557166 \\
\hline $\mathrm{H}$ & 4.7633374335 & 7.8716201868 & 15.3532209752 \\
\hline $\mathrm{Cd}$ & 8.2578845764 & 4.7032414935 & 13.0478380627 \\
\hline $\mathrm{Cl}$ & 10.2625384061 & 2.1980117814 & 4.5575189251 \\
\hline $\mathrm{Cl}$ & -1.2417999213 & 8.5439627425 & 4.6606601110 \\
\hline
\end{tabular}

\subsubsection{Coordinates of $\mathrm{g} 6 \times 6 / 3 \mathrm{~d}-\mathrm{Hg}^{2+}, 2 \mathrm{Cl}^{-}$}


90

\begin{tabular}{|c|c|c|c|}
\hline Y & -1.4020049800 & -0.9454811007 & 7.6797584605 \\
\hline C & -1.4052191910 & 0.4785259377 & 7.6850173367 \\
\hline & -2.6380881376 & 1.1908436056 & 7.6763493580 \\
\hline & -2.6399090631 & 2.6141515703 & 7.6916594080 \\
\hline & -3.8703103456 & 3.3274319805 & 7.6699796138 \\
\hline & -3.8685579320 & 4.7516386683 & 7.6730489122 \\
\hline & -5.1033336021 & 5.4642730189 & 7.6510004320 \\
\hline & -5.1023552934 & 6.8881036349 & 7.6504273586 \\
\hline & -6.3385868139 & 7.6002551763 & 7.6585259922 \\
\hline & -6.3365485895 & 9.0259972063 & 7.6585758082 \\
\hline & -7.5682802662 & 9.7389369861 & 7.6777852707 \\
\hline & -7.5702647139 & 11.1630707366 & 7.6746287563 \\
\hline & 1.0656680182 & -0.9438584516 & 7.6809099306 \\
\hline & 1.0630103181 & 0.4793576036 & 7.7188797464 \\
\hline & -0.1712733875 & 1.1893730303 & 7.7173824239 \\
\hline & -0.1759586500 & 2.6117637767 & 7.7672978443 \\
\hline & -1.4058435256 & 3.3259285606 & 7.7481169808 \\
\hline & -1.4024678081 & 4.7492601137 & 7.7867055761 \\
\hline & -2.6353881027 & 5.4644369027 & 7.7215573938 \\
\hline & -2.6345128289 & 6.8894987478 & 7.7189673894 \\
\hline & -3.8672647938 & 7.6006868299 & 7.6671455865 \\
\hline & -3.8680515362 & 9.0262447181 & 7.6631671179 \\
\hline & -5.1018880617 & 9.7386003788 & 7.6538163950 \\
\hline & -5.1026718429 & 11.1632934568 & 7.6690186490 \\
\hline & 3.5333385971 & -0.9440769912 & 7.6754695991 \\
\hline & 3.5347314784 & 0.4789445834 & 7.7116993575 \\
\hline & 2.2991706930 & 1.1955741260 & 7.7601957390 \\
\hline & 2.2995958592 & 2.6100734720 & 7.8597448537 \\
\hline & 1.0552168550 & 3.3229177095 & 7.8769949154 \\
\hline & 1.0360005585 & 4.7260460434 & 8.0225492305 \\
\hline & -0.1678909430 & 5.4540966632 & 7.9239564459 \\
\hline & -0.1692638531 & 6.9011768084 & 7.9158598175 \\
\hline & -1.4016171739 & 7.6042831911 & 7.7782597870 \\
\hline & -1.4037894306 & 9.0283650300 & 7.7315447961 \\
\hline & -2.6370361812 & 9.7394023155 & 7.6826137806 \\
\hline & -2.6357859729 & 11.1624420564 & 7.6688457104 \\
\hline
\end{tabular}




\begin{tabular}{|c|c|c|c|}
\hline C & 5.9990948677 & -0.9452824207 & 7.6691664697 \\
\hline C & 6.0014303048 & 0.4783131978 & 7.6676724089 \\
\hline C & 4.7678378329 & 1.1903273878 & 7.7016025972 \\
\hline C & 4.7716653494 & 2.6131768829 & 7.7495137979 \\
\hline C & 3.5420586440 & 3.3254551591 & 7.8738398932 \\
\hline C & 3.5652328518 & 4.7293190821 & 8.0292966925 \\
\hline C & 2.3003521091 & 5.4075655719 & 8.3753819983 \\
\hline C & 2.2955751916 & 6.9487472915 & 8.3378074197 \\
\hline C & 1.0366351792 & 7.6283114864 & 7.9921893892 \\
\hline C & 1.0554863075 & 9.0316320545 & 7.8448485779 \\
\hline C & -0.1751606504 & 9.7425273979 & 7.7461653778 \\
\hline C & -0.1703146141 & 11.1638010746 & 7.6963329859 \\
\hline C & 8.4656783954 & -0.9459564092 & 7.6952605935 \\
\hline C & 8.4682094225 & 0.4766442383 & 7.6624727414 \\
\hline C & 7.2347588437 & 1.1905803022 & 7.6598050892 \\
\hline C & 7.2358433852 & 2.6141612515 & 7.6710511083 \\
\hline C & 6.0011213042 & 3.3265203445 & 7.7201139334 \\
\hline C & 5.9965303980 & 4.7500581449 & 7.7485342508 \\
\hline C & 4.7649427437 & 5.4533519482 & 7.8931675580 \\
\hline C & 4.7649748235 & 6.8971345922 & 7.9022602684 \\
\hline C & 3.5620136984 & 7.6233309991 & 8.0152099253 \\
\hline C & 3.5409823879 & 9.0288149647 & 7.8599332612 \\
\hline C & 2.2986107554 & 9.7438240880 & 7.8321717587 \\
\hline C & 2.2989129999 & 11.1587786570 & 7.7373422285 \\
\hline C & 10.9358993661 & -0.9451770898 & 7.7018066071 \\
\hline C & 10.9335766207 & 0.4768476216 & 7.6708631609 \\
\hline C & 9.7006990099 & 1.1894332466 & 7.6571496972 \\
\hline C & 9.7002195454 & 2.6147872370 & 7.6500819612 \\
\hline C & 8.4676451027 & 3.3265772978 & 7.6581998017 \\
\hline C & 8.4651697239 & 4.7521074467 & 7.6570622648 \\
\hline C & 7.2314585199 & 5.4634042919 & 7.6927056978 \\
\hline C & 7.2318837337 & 6.8890415508 & 7.6972446332 \\
\hline C & 5.9981638275 & 7.6029750130 & 7.7603619699 \\
\hline C & 6.0010748747 & 9.0260239298 & 7.7269943261 \\
\hline C & 4.7711284117 & 9.7405309470 & 7.7486843463 \\
\hline C & 4.7670178976 & 11.1637455260 & 7.7011983721 \\
\hline C & 2.2016935716 & 6.1843009785 & 9.7079901756 \\
\hline
\end{tabular}




$\begin{array}{lrrr}\mathrm{C} & 3.2320109636 & 6.2455717231 & 10.7820490048 \\ \mathrm{H} & 1.1780653266 & 6.2066209679 & 10.0781579246 \\ \mathrm{C} & 2.7959406016 & 6.8197145653 & 12.0976264049 \\ \mathrm{O} & 4.3898025278 & 5.8470793979 & 10.6325094605 \\ \mathrm{C} & 3.5994814959 & 6.5371670017 & 13.2163404463 \\ \mathrm{C} & 3.2868824088 & 7.0671925366 & 14.4636161874 \\ \mathrm{C} & 2.1748929342 & 7.9031845403 & 14.6090584977 \\ \mathrm{C} & 1.3760035734 & 8.2028897393 & 13.5029199673 \\ \mathrm{C} & 1.6774962068 & 7.6573098897 & 12.2536850337 \\ \mathrm{H} & 1.0498289694 & 7.9128075339 & 11.3982290294 \\ \mathrm{H} & 1.9339966558 & 8.3222300859 & 15.5865495276 \\ \mathrm{H} & 0.5157870161 & 8.8641971099 & 13.6172051972 \\ \mathrm{H} & 4.4682744827 & 5.8935763744 & 13.0753513157 \\ \mathrm{H} & 3.9100928880 & 6.8323344894 & 15.3274126628 \\ \mathrm{Hg} & 6.7666421623 & 3.2440489241 & 12.1514384067 \\ \mathrm{Cl} & 10.2187513058 & 2.1874601421 & 4.5389786900 \\ \mathrm{Cl} & -1.2657686130 & 8.5415095994 & 4.6026131323\end{array}$




\section{Electron density difference plots}

g6 $66 / 2 a$

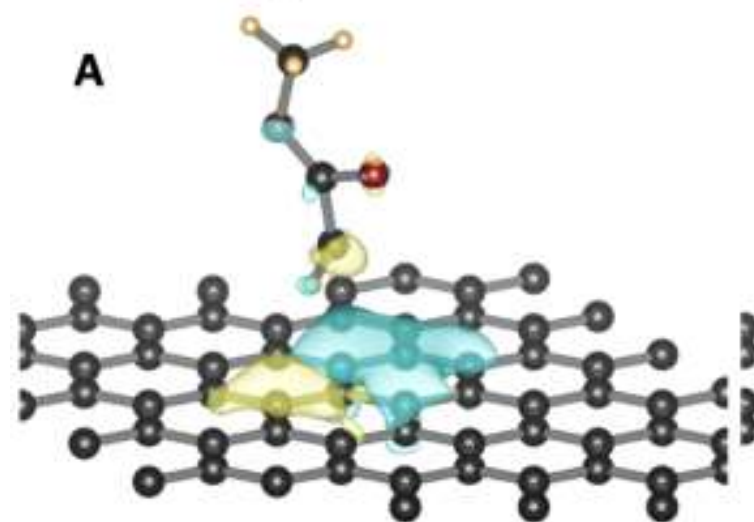

B
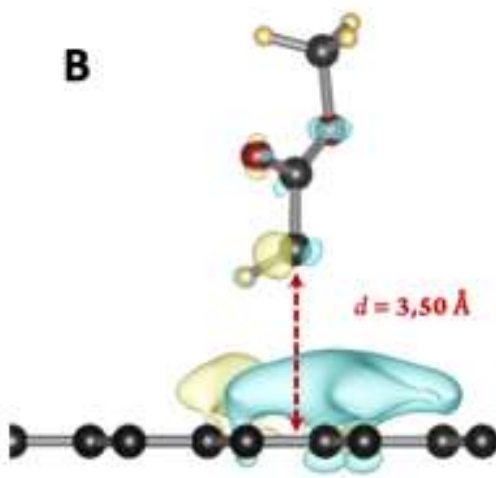

$\mathrm{g} 6 \times 6 / 2 \mathrm{c}$

C

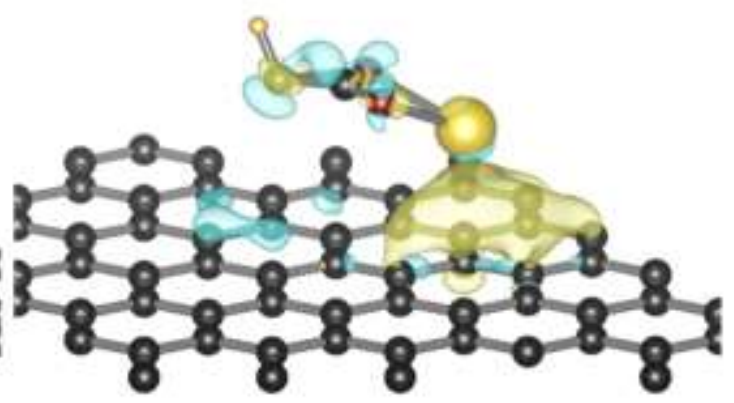

D

Figure S13. Isocontour plots of electron density differences for the systems $(\mathbf{g 6} \times \mathbf{6} / \mathbf{2 a})$ and $(\mathbf{g 6} \times \mathbf{6} / \mathbf{2 c})$ before and after functionalization, showing regions of increase in electron density () and lodd of electron density (light blue). Values of isocontour are fixed to $2.0 \times 10^{-4} \mathrm{e}^{-\mathrm{Bohr}^{3}}$ and $1,0 \times 10^{-3} \mathrm{e} \mathrm{Bohr}^{3}$ for the systems $($ g6 $\times 6 / 2 a)$ and $(\mathbf{g 6} \times 6 / 2 c)$, respectively. 